\title{
Single-Commodity Robust Network Design with Finite and Hose Demand Sets*
}

\author{
Valentina Cacchiani ${ }^{1}$, Michael Jünger ${ }^{2}$, Frauke Liers ${ }^{3}$, Andrea Lodi ${ }^{1}$, \\ and Daniel R. Schmidt ${ }^{2}$ \\ ${ }^{1}$ DEI, University of Bologna, Viale Risorgimento 2, I-40136, Bologna, Italy, \\ \{valentina.cacchiani, andrea.lodi\}@unibo.it \\ ${ }^{2}$ Institut für Informatik, Universität zu Köln, Albertus-Magnus-Platz, 50923 Köln, Germany, \\ \{mjuenger, schmidt\}@informatik. uni-koeln.de \\ ${ }^{3}$ Department Mathematik, Friedrich-Alexander Universität Erlangen-Nürnberg, Cauerstraße 11, 91058 \\ Erlangen, Germany, frauke.liers@math.uni-erlangen.de
}

March 17th, 2014

This preprint also appeared as technical report OR-14-11 at the University of Bologna.

We study a single-commodity Robust Network Design problem (sRND) defined on an undirected graph. Our goal is to determine minimum cost capacities such that any traffic demand from a given uncertainty set can be satisfied by a feasible single-commodity flow. We consider two ways of representing the uncertainty set, either as a finite list of scenarios or as a polytope. We propose a branch-andcut algorithm to derive optimal solutions to SRND, built on a capacity-based integer linear programming formulation. It is strenghtened with valid inequalities derived as $\left\{0, \frac{1}{2}\right\}$-Chvátal-Gomory cuts. Since the formulation contains exponentially many constraints, we provide practical separation algorithms. Extensive computational experiments show that our approach is effective, in comparison to existing approaches from the literature as well as to solving a flow based formulation by a general purpose solver.

\footnotetext{
${ }^{*}$ We thank the Ateneo Italo-Tedesco VIGONI programme 2011-2012 for its financial support.
} 


\section{Introduction}

We consider a single-commodity network design problem (sRND) that is robust in the sense of Ben-Tal and Nemirowski [11]: Given an undirected graph $G=(V, E)$ and an (bounded) uncertainty set of traffic demands $\mathfrak{D} \subseteq \mathbb{R}^{V}$, we can install multiples of a unit capacity on the edges of $G$. Installing one unit of capacity on $e \in E$ incurs a cost of $c_{e}$. The goal is to find minimum cost capacities $u$ such that for all traffic demand vectors $b \in \mathfrak{D}$, there exists a feasible directed single-commodity flow in $(G, u)$ that routes $b$. Problems of this type arise in the design process of different kind of networks, e.g., transportation and telecommunication networks, but also in energy and gas distribution planning. As even small changes in the traffic demands can cause congestion and network failures, practical solutions should be robust against traffic demands that fluctuate over time or cannot be known with arbitrary precision. This is where robust network design comes in.

Most network design models go back to a problem definition by Gomory and $\mathrm{Hu}$ [26] where there is a traffic requests $r_{i j}$ for each pair $i, j$ of nodes and $r_{i j}$ units of flow have to be sent from $i$ to $j$. This means that the underlying flow model is a multi-commodity flow and that the assignment of sources to sinks is fixed, which is not always desirable. Imagine for example a network where several identical servers can answer all the traffic requests of the clients: There, cheaper solutions can be obtained when the optimization process is allowed to map clients to servers instead of using a fixed mapping from the problem input. In that case, the underlying flow model should be a single-commodity flow. One example of such networks would be a movie streaming network [16] or a network of servers for mirrored software distribution. Gas and energy distribution networks also ship a single commodity, but since we do not want to model the complex physical properties of these networks here, we focus on communication networks as our application.

The above single-commodity model is due to Buchheim, Liers and Sanità [41, 16] who additionally assume that $\mathfrak{D}$ is a finite set in the spirit of Minoux [36]. They propose an integer linear programming formulation that is based on arc-flow variables and strengthen it with certain general cutting planes called target cuts. The model allows to compute a different routing for each $b \in \mathfrak{D}$. We call this way of routing a dynamic routing, as opposed to static routing schemes that route all scenarios on the same fixed set of paths. As a consequence, one set of arc-flow variables is needed for each $b \in \mathfrak{D}$. In a previous joint work [4] with Álvarez-Miranda and Parriani, the authors of this article present a linear programming based heuristic for this model. Here, however, we are interested in solving the problem with an exact algorithm and show a different integer programming formulation whose size does not depend on $|\mathfrak{D}|$. Parts of these results appeared in [3] as an extended abstract. Our alternative formulation is based on cut-set and 3-partition inequalities. Both types originally appeared in non-robust network design, see [31] for the original application of these inequalities and $[9,15,14]$ for examples of advanced cut-set based branch-and-cut algorithms. Additionally, Atamtürk [6] and Raack, Koster and Wessäly [40] give extensive surveys of the use of these inequalities in network design. Avello, Mattia and Sassano [7] derive a branch-and-cut algorithm for robust multi-commodity network design with 
a finite demand set that is based on the more general metric inequalities.

Moreover, we apply the robustification approach by Ben-Tal and Nemirowski [11] to the above model. In their approach, the uncertainty set $\mathfrak{D}$ is given by a polytope in a linear description. Several prior applications of the approach to multi-commodity network design exist and many of them are again succesfully using cut-set inequalities: Ben-Ameur and Kerivin [10] consider the multicommodity network design problem by [26] with a general demand polytope and static routing. There is an extension to dynamic routing by Mudchanatongsuk, Ordóñez and Liu [37]. Koster, Kutschka and Raack apply the $\Gamma$-robustness approach by Bertsimas and Sim [13], using again static routing. Altın, Amaldi, Belotti and Pinar instead consider the Hose uncertainty model that was proposed by Fingerhut, Suri and Turner [23] and Duffield et al. [22]. They also consider a combination with the $\Gamma$-robustness model. Mattia [34] considers the Hose model with dynamic routing. We show a natural adaption of the Hose model to singlecommodity flows in the model by Buchheim et al. [16] and solve it again with a cut-set model. Pesenti, Rinaldi and Ukovich [39] solve the related Minimum Cost Network Containment problem using cut-set inequalities (see Section 4).

Our contribution. In this paper, we consider the sRND problem and distinguish two ways of representing the uncertainty set $\mathfrak{D}$ in the input: it can be given as a finite list of scenarios or as a linear description of a polytope. Our goal is to determine optimum solutions for the sRND by providing an effective branch-and-cut algorithm in both cases. To this aim, we present a capacity-based ILP formulation. The formulation was introduced in [3] for the case of finite scenario list and uses cut-set inequalities. We prove that the corresponding polyhedron is full dimensional and define the conditions under which a cut-set inequality defines a facet. The size of the model depends only on the size of the network, but not on the number of scenarios, as opposed to the flow-based model by Buchheim et al. [16]. On the other hand, it contains exponentially many constraints. We provide a polynomial time algorithm for the separation of cut-set inequalities for the case that $\mathfrak{D}$ is finite. We prove that the separation problem is NP-hard when $\mathfrak{D}$ is given as a polytope, even when $\mathfrak{D}$ is based on an adaption of the Hose model [22]. Still, in this case we propose a practical separation algorithm using a simple mixed integer program (MIP). We strengthen our formulation with 3-partition inequalities and show how to separate them as $\left\{0, \frac{1}{2}\right\}$-Chvátal-Gomory cuts, as defined by [17]. Extensive computational experiments show that our approach is effective, in comparison to existing approaches from the literature as well as to solving a flow based formulation by a general purpose solver.

General notation and problem definition. For $a \in \mathbb{R}^{n}, b \in \mathbb{R}^{m}$ and $n, m \in \mathbb{N}$, we say that $a^{T} x^{*}-b$ is the slack of the inequality $a^{T} x \geq b$ with respect to a vector $x^{*} \in \mathbb{R}^{n}$. We say that $a^{T} x \geq b$ is binding for $x^{*}$ if its slack with respect to $x^{*}$ is zero and we say that $a^{T} x \geq b$ is violated by $x^{*}$ if its slack with respect to $x^{*}$ is negative.

Given an undirected graph $G=(V, E)$ with capacities $u: E \rightarrow \mathbb{Z}_{\geq 0}$ and a node balance vector $b \in \mathbb{R}^{V}$, a directed, single-commodity $b$-flow is commonly defined as a directed flow $f \in \mathbb{R}^{E}$ 
that satisfies the following two conditions.

1. For all nodes $i \in V$, we require that $\sum_{j \in \delta(i)}\left(f_{i j}-f_{j i}\right)=b_{i}$ and call this condition the flow balance condition.

2. For all edges $\{i, j\} \in E$, we have $f_{i j}+f_{j i} \leq u_{i j}$. We also say that $f$ respects $u$.

Using this definition we can define the Single-Commodity Robust Network Design Problem (sRND) as follows. The input is an undirected graph $G=(V, E)$, an uncertainty set $\mathfrak{D} \subseteq \mathbb{R}^{V}$ of balance vectors and a cost function $c: E \rightarrow \mathbb{R}$ such that installing one unit of capacity on edge $e$ costs $c_{e}$. The task is to determine integral capacities $u: E \rightarrow \mathbb{Z}_{>0}$ such that for all balance vectors $b \in \mathfrak{D}$ there exists a directed single-commodity $b$-flow in $G$ that satisfies the capacity conditions with respect to $u$ and minimizes the total capacity installation $\operatorname{cost} \sum_{e \in E} c_{e} u_{e}$.

Thus, we need to design a network that supports a certain $b$-flow, but due to uncertain information, we cannot know $b$ exactly. Therefore, we create a set $\mathfrak{D}$ that contains all possible realizations of $b$ and guarantee that no matter what $b \in \mathfrak{D}$ is actually realized, we can route it. We refer to the vectors in $\mathfrak{D}$ as scenarios for this reason. If $\mathfrak{D}$ finite, we call the corresponding problem a finite sRND problem. If $\mathfrak{D}$ is a polytope, we refer to the underlying problem as the polytopal sRND problem.

Finite Versus Polyhedral Uncertainty Sets. The finite and the polytopal sRND problem are equivalent in the following sense: Any finite uncertainty set can be replaced by the polytope defined by its convex hull and any polytopal (i.e., bounded) uncertainty set can be replaced by the finite set of its vertices. Both reductions do not change the set of feasible flows. However, they do change the size of the problem input. In general, its size can grow exponentially (see Section 5). Therefore, in any given application, the suitable model needs to be chosen carefully: The finite sRND model should be preferred when the extreme points of the uncertainty set are known and if their number is small. On the other hand, the polytopal model is suited better when the uncertainty set has a small linear description. Thus, we will consider both cases in the scope of this article in spite of their apparent equivalence. Nonetheless, all results that we prove for finite scenario sets also hold for a polytopal scenario set (and vice-versa), as far as they do not concern computational complexity.

Organisation of the article. The paper is organized as follows. In Section 2 we present results that concern both the finite and the polytopal case. Specialized results for both cases follow in Section 3 and Section 4, respectively. We conclude in Section 5 with a branch-and-cut algorithm and computational results.

\section{Integer Programming Formulations and Polyhedral Results}

We start our considerations with results that concern both the finite and the polyhedral case. 


\subsection{A Capacity-Based Integer Programming Formulation}

In order to obtain a cut-based formulation for the sRND problem, consider some subset $S \subseteq V$ of a graph's node set. We denote the set of edges that have one end-node in $S$ by $\delta(S)$ and call $\delta(S)$ an (edge) cut in $G$. Consequently, we also call $S$ a cut-set. We can compute the maximum total balance of $S$ as $R_{S}:=\max _{b \in \mathscr{D}}\left|\sum_{i \in S} b_{i}\right|$ and we observe that $R_{S}$ is exactly the amount of flow that cannot be balanced out within $S$. At least $R_{S}$ units of flow must cross the cut $\delta(S)$ and therefore, for any $S \subseteq V$, the capacity of $\delta(S)$ must be at least $R_{S}$. This gives rise to the concept of a cut-set inequality.

Definition 1. Let $G=(V, E)$ be an undirected graph, let $S \subseteq V$ and assume that $\mathfrak{D}$ is a finite or a polyhedral uncertainty set. We then call the inequality

$$
\sum_{\{i, j\} \in \delta(S)} u_{i j} \geq \max _{b \in \mathfrak{D}}\left|\sum_{i \in S} b_{i}\right|
$$

the cut-set-inequality induced by $S$. We use $\left(C S_{S}\right)$ as a short-hand notation for the inequality and we denote its right hand side by $R_{S}$.

Writing down the cut-set inequalities for all node subsets, we obtain the following integer linear programming problem that will turn out to be a cut-based formulation for the sRND problem:

$$
\begin{array}{cll}
\min & \sum_{\{i, j\} \in E} c_{i j} u_{i j} & \\
\text { s.t. } & \sum_{\{i, j\} \in \delta(S)} u_{i j} \geq \max _{b \in \mathfrak{D}}\left|\sum_{i \in S} b_{i}\right| & \text { for all } S \subseteq V \\
& u_{i j} \in \mathbb{Z}_{\geq 0} & \text { for all }\{i, j\} \in E
\end{array}
$$

Denote by $\mathfrak{P}_{\mathrm{sRND}}^{f}(G, \mathfrak{D})$ the set of all, possibly fractional, capacity vectors $u \in \mathbb{R}_{>0}^{E}$ that permit sending a $b$-flow on $G$ for all scenarios $b \in \mathfrak{D}$ and by $\mathfrak{P}_{\mathrm{sRND}}(G, \mathfrak{D})$ the convex hull of all integer points in $\mathfrak{P}_{\text {sRND }}^{f}(G, \mathfrak{D})$ (both sets are unbounded). Then, the linear programming relaxation of the cut-set formulation (IP-CS) exactly describes $\mathfrak{P}_{\mathrm{sRND}}^{f}(G, \mathfrak{D})$, as we show in Theorem 2.

Theorem 2. A vector $u \in \mathbb{R}_{\geq 0}^{E}$ is feasible for the linear programming relaxation of the integer program (IP-CS) if and only if there exists a feasible b-flow that respects the capacities $u$ in $G$ for all scenarios $b \in \mathfrak{D}$.

Proof. For the first part, let $u \in \mathfrak{P}_{\mathrm{sRND}}^{f}(G, \mathfrak{D})$. We consider an arbitrary subset $S \subseteq V$ of the nodes and any scenario $b \in \mathfrak{D}$. By our assumption, there exists a feasible $b$-flow $f$ on $G$ that respects $u$. Adding up the flow-conservation conditions for all $i \in S$ yields the well-known result 
that:

$$
\begin{aligned}
\left|\sum_{i \in S} b_{i}\right| & =\left|\sum_{i \in S} \sum_{j \in \delta(i)}\left(f_{i j}-f_{j i}\right)\right|=|\underbrace{\mid \sum_{i \in S} \sum_{j \in \delta(i) \cap S}\left(f_{i j}-f_{j i}\right)}_{=0}+\sum_{i \in S} \sum_{j \in \delta(i) \cap V \backslash S}\left(f_{i j}-f_{j i}\right)| \\
& \left.=\left|\sum_{\{i, j\} \in \delta(S)}\left(f_{i j}-f_{j i}\right)\right|^{f \geq 0}|\underset{\{i, j\} \in \delta(S)}{\leq}| f_{i j}+f_{j i}\right) \mid \leq \sum_{\{i, j\} \in \delta(S)} u_{i j} .
\end{aligned}
$$

This shows that $u$ satisfies all cut-set inequalities.

On the other hand, let $u$ be a feasible vector for (IP-CS) and let again $b \in \mathfrak{D}$ be some arbitrary scenario. To show that $u \in \mathfrak{P}_{\mathrm{sRND}}^{f}(G, \mathfrak{D})$, we need to show that for every $b \in \mathfrak{D}$, there exists a feasible $b$-flow under $u$. Yet, iff for some $b \in \mathfrak{D}$, no feasible $b$-flow exists in $(G, u)$, then by the MaxFlow-MinCut theorem [24] there exists some cut $S$ with a capacity $\sum_{\{i, j\} \in \delta(S)} u_{i j}$ strictly less than $\left|\sum_{i \in S} b_{i}\right|$. This is a contradiction to the assumption that $u$ satisfies all cut-setinequalities.

In particular, the (non-relaxed) cut-set formulation (IP-CS) exactly characterizes all (infinitely many) integral points in $\mathfrak{P}_{\mathrm{sRND}}(G, \mathfrak{D})$.

Corollary 3. A capacity vector $u \in \mathbb{Z}_{>0}^{E}$ is feasible for the $s R N D$ instance $(G, \mathfrak{D})$ if and only if it is feasible for the cut-set formulation (IP-CS).

The exponential size of the cut-set formulation (IP-CS) naturally raises the question of cutset constraint separation which we postpone to Sections 3 and 4. To conclude this section, we state that $\mathfrak{P}_{\mathrm{sRND}}(G, \mathfrak{D})$ has full dimension and that the cut-set inequalities are facet-defining for $\mathfrak{P}_{\mathrm{sRND}}(G, \mathfrak{D})$. Both results were already known for the non-robust multi-commodity network design problem [31] since the 90's, before Mattia [34] gave a much shorter proof for the mRND in 2010. Finally, Dorneth observed in his diploma thesis [21] that Mattia's proofs only need small adaptations for the finite sRND. We repeat a more concise version here in order to make the adapted proofs available and to extend them to the polyhedral demand case. As before we define $R_{S}:=\max _{b \in \mathfrak{D}}\left|\sum_{i \in S} b_{i}\right|$. We let $B^{*}:=\max _{b \in \mathfrak{D}} \sum_{i \in V}\left|b_{i}\right|$ be an upper bound for the capacity on any edge.

Theorem 4 ([34, 21]). For any network $G=(V, E)$ and any (finite or polyhedral) demand set $\mathfrak{D}$, the polyhedron $\mathfrak{P}_{\mathrm{sRND}}(G, \mathfrak{D})$ is full dimensional, i.e. its dimension is $|E|$.

Proof. We define a capacity vector $u^{e}$ for every edge $e \in E$ in the following way:

$$
u_{e^{\prime}}^{e}:=\left\{\begin{array}{ll}
B^{*}+1, & \text { if } e^{\prime}=e \\
B^{*}, & \text { otherwise }
\end{array} \quad \text { for all } e^{\prime} \in E\right.
$$

and additionally define $U \equiv B^{*}$ as the capacity vector whose entries are all equal to $B^{*}$. Since we have to install at most a capacity of $B^{*}$ on any edge, we have $U \in \mathfrak{P}_{\mathrm{sRND}}(G, \mathfrak{D})$ and $u^{e} \in$ $\mathfrak{P}_{\mathrm{sRND}}(G, \mathfrak{D})$ for all $e \in E$. Also, we obtain $|E|$ distinct unit vectors by looking at $u^{e}-U$ for all $e \in E$. Thus, $\operatorname{dim}\left(\mathfrak{P}_{\mathrm{sRND}}(G, \mathfrak{D})\right)=|E|$. 
Theorem 5 ([34, 21]). For any cut $S \subseteq V,\left(C S_{S}\right)$ defines a facet of $\mathfrak{P}_{\mathrm{sRND}}(G, \mathfrak{D})$ if and only if $R_{S}>0$ and the subgraphs induced by $S$ and $V \backslash S$ are connected.

Proof. If $R_{S}=0,\left(\mathrm{CS}_{S}\right)$ cannot be stronger than the trivial inequalities $u_{e} \geq 0$ for $e \in \delta(S)$. Also, if $S$ (or likewise, $V \backslash S$ ) decomposes into several connected components $S_{1}, \ldots, S_{k}$, then summing up the inequalities we get from $S_{1}, \ldots, S_{k}$ yields the same left hand side as we get from $S$; yet, the right-hand side of $\left(\mathrm{CS}_{S_{1}}\right)+\cdots+\left(\mathrm{CS}_{S_{k}}\right)$ can only be stronger than the one of $\left(\mathrm{CS}_{S}\right)$ by the triangle inequality.

Finally, in order to show that $\left(\mathrm{CS}_{S}\right)$ defines a facet of $\mathfrak{P}_{\mathrm{sRND}}(G, \mathfrak{D})$ we define a vector $u^{e}$ for every edge $e \in E$ in the way suggested by Mattia [34, Theorem 3.14]. In doing so, our choice depends on whether $e$ lies in $\delta(S)$. For all $e \in \delta(S)$, define $u^{e}$ as

$$
u_{e^{\prime}}^{e}:=\left\{\begin{array}{ll}
R_{S} & \text { if } e^{\prime} \in \delta(S), e^{\prime}=e \\
0 & \text { if } e^{\prime} \in \delta(S), e^{\prime} \neq e \\
B^{*} & \text { if } e^{\prime} \notin \delta(S)
\end{array} \quad \text { for all } e^{\prime} \in E\right.
$$

Now, for all $e \notin \delta(S)$ and some fixed $h \in \delta(S)$ choose $u^{e}$ as

$$
u_{e^{\prime}}^{e}:=\left\{\begin{array}{ll}
R_{S} & \text { if } e^{\prime} \in \delta(S), e^{\prime}=h \\
0 & \text { if } e^{\prime} \in \delta(S), e^{\prime} \neq h \\
B^{*}+1 & \text { if } e^{\prime} \notin \delta(S), e^{\prime}=e \\
B^{*} & \text { if } e^{\prime} \notin \delta(S), e^{\prime} \neq e
\end{array} \quad \text { for all } e^{\prime} \in E\right.
$$

Because we have $R_{S} \neq 0$, the vectors $u^{e}, e \in E$, are linearly independent. This is easily verfified by considering the upper triangular matrix with the rows $u^{e}$ for $e \in \delta(S)$ followed by the rows $u^{e}-u^{h}$ for $e \notin \delta(S)$. For all $e \in \delta(S)$, the vector $u^{e}$ satisfies $\left(\mathrm{CS}_{S}\right)$ with equality since $\sum_{e^{\prime} \in \delta(S)} u_{e^{\prime}}^{e}=u_{e}^{e}=R_{S}$ by the definition of $u^{e}$. If $e \notin \delta(S)$, we have instead $\sum_{e^{\prime} \in \delta(S)} u_{e^{\prime}}^{e}=u_{h}^{e}=R_{S}$ and again, $\left(\mathrm{CS}_{S}\right)$ is satisfied with equality.

It remains to show that $u^{e} \in \mathfrak{P}_{\mathrm{sRND}}(G, \mathfrak{D})$ for all $e \in E$. We fix an arbitrary cut $X \subseteq V$ such that the subgraphs $G[X]$ and $G[V \backslash X]$ which are induced by $X$ and $V \backslash X$, respectively, are connected and show that $u^{e}$ satisfies $\left(\mathrm{CS}_{X}\right)$ for all $e \in E$. We can assume that $X \neq S$ and that $X \neq V \backslash S$ since we have already shown validity for those two cases. Thus, if $\delta(X) \subseteq \delta(S)$ was true, then either $G[X]$ or $G[V \backslash X]$ would not be connected and therefore, there exists at least one edge $e^{*} \in \delta(X) \backslash \delta(S)$. Using this observation for any $e \in E$ we have

$$
\sum_{e^{\prime} \in \delta(X)} u_{e^{\prime}}^{e} \geq \sum_{e^{\prime} \in \delta(X) \backslash \delta(S)} u_{e^{\prime}}^{e} \geq u_{e^{*}}^{e} \geq B^{*}=\max _{b \in \mathfrak{D}} \sum_{i \in V}\left|b_{i}\right| \geq \max _{b \in \mathfrak{D}}\left|\sum_{i \in X} b_{i}\right|
$$

which tells us that $u^{e}$ satisfies $\left(\mathrm{CS}_{X}\right)$. We conclude that $\left(\mathrm{CS}_{S}\right)$ defines a face of dimension $|E|-1$ and, therefore, is a facet of $\mathfrak{P}_{\mathrm{sRND}}(G, \mathfrak{D})$. 


\subsection{Valid 3-Partition Inequalities Derived from Chvátal-Gomory Cuts}

The cut-set inequalities $\left(\mathrm{CS}_{S}\right)$ give a lower bound on the amount of capacity that is needed along the cut that separates a 2-partition $S \subseteq V$ and $V \backslash S$. In general, however, one can ask for lower bounds on the capacity between any $k$-partition, $k \geq 2$, of the graph. This leads to the definition of $k$-partition inequalities, an idea that was e.g. explored by [1]. We will see that 3-partition inequalities can be separated as $\left\{0, \frac{1}{2}\right\}$-Chvátal-Gomory cuts as defined by [17] and elaborate on the details in this subsection. A similar result has been obtained by Magnanti, Michandani and Vachani [32] for a non-robust multi-commodity network design problem.

For any given linear program $A x \geq b$ with a constraint matrix $A=\left(a_{i j}\right) \in \mathbb{Z}^{m \times n}$ and vectors $x \in \mathbb{R}^{n}, b \in \mathbb{Z}^{m}$ one can generate a valid inequality for $A x \geq b$ by selecting some subset $I \subseteq$ $\{1, \ldots, m\}$ of the constraints and computing the inequality $\frac{1}{2} \cdot \sum_{j=1}^{n} \sum_{i \in I} a_{i j} x_{j} \geq \frac{1}{2} \sum_{i \in I} b_{i}$. If the coefficients $\sum_{i \in I} \frac{1}{2} a_{i j}$ are integral for all $j=1, \ldots, n$, we can round up the right hand side of the inequality and thus obtain a $0-\frac{1}{2}$-cut [17]. The problem is, of course, to select a suitable set $I$ that generates integral coefficients. Due to the structure of the cut-set inequalities, we can solve this problem if we restrict to $|I|=3,4$. Indeed, observe that for two non-empty sets $S, T \subsetneq V$ and any vector $u \in \mathbb{R}_{\geq 0}^{E}$, we have by a counting argument

$$
\sum_{e \in \delta(S)} u_{e}+\sum_{e \in \delta(T)} u_{e}+\sum_{e \in \delta(S \cup T)} u_{e}+\sum_{e \in \delta(S \cap T)} u_{e}=2 \sum_{e \in \delta(S \cup T)} u_{e}+2 \sum_{e \in(S: T)} u_{e}+2 \sum_{e \in \delta(S \cap T)} u_{e} .
$$

where $(S: T)$ is defined as the set of edges $\delta(S) \cap \delta(T)$ having one end node in $S$ and one end node in $T$. Therefore, given cut-set inequalities $\left(\mathrm{CS}_{S}\right)$ and $\left(\mathrm{CS}_{T}\right)$, we obtain a valid zero-half cut by adding up $\frac{1}{2}\left(\left(\mathrm{CS}_{S}\right)+\left(\mathrm{CS}_{T}\right)+\left(\mathrm{CS}_{S \cup T}\right)+\left(\mathrm{CS}_{S \cap T}\right)\right)$ to

$$
\sum_{e \in \delta(S \cup T)} u_{e}+\sum_{e \in(S: T)} u_{e}+\sum_{e \in \delta(S \cap T)} u_{e} \geq\left\lceil\frac{1}{2}\left(R_{S}+R_{T}+R_{S \cup T}+R_{S \cap T}\right)\right\rceil .
$$

If $R_{S}+R_{T}+R_{S \cup T}+R_{S \cap T}$ is odd, the violation of $\left(\mathrm{ZH}_{S, T}\right)$ with respect to a solution $u^{*}$ is maximum if $\left(\mathrm{CS}_{S}\right),\left(\mathrm{CS}_{T}\right),\left(\mathrm{CS}_{S \cup T}\right)$ and $\left(\mathrm{CS}_{S \cap T}\right)$ are binding for $u^{*}$. Therefore, we should select sets $S, T$ where the corresponding cut-set inequalities have small slack.

This observation implies a simple separation algorithm EnumZH: We iterate over all pairs $\left(\mathrm{CS}_{S}\right),\left(\mathrm{CS}_{T}\right)$ of binding cut-set inequalities in our constraint set. We then build the corresponding zero-half cut $\left(\mathrm{ZH}_{S, T}\right)$ and check if it is violated. The running time of the algorithm is quadratic in the number of binding cut-set constraints. While these can be exponentially many (see Figure 1), our experiments show that it pays off to use the algorithm at the root node of the branch and cut tree, see Section 5.

We can replace $\left(\mathrm{CS}_{S \cup T}\right)$ by $\left(\mathrm{CS}_{V \backslash(S \cup T)}\right)$ in the above construction without changing $\left(\mathrm{ZH}_{S, T}\right)$. Then, if $S$ and $T$ are disjoint sets, an edge $e \in E$ has a non-zero coefficient in $\left(\mathrm{ZH}_{S, T}\right)$ if and only if it is contained in $(S: T),(S: V \backslash(S \cup T))$ or $(T: V \backslash(S \cup T))$. Thus, $\left(\mathrm{ZH}_{S, T}\right)$ defines a 3-partition inequality for the partitions $S, T$ and $V \backslash(S \cup T)$. In this way, EnumZH is a separation heuristic for 3-partition inequalities. 


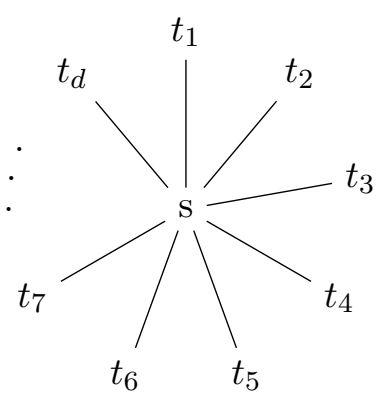

Figure 1: An instance that has a high number of binding cut-set inequalities at the optimum. We define a single scenario: node $s$ has a supply of $d$ and all other nodes $t_{1}, \ldots, t_{d}$ have a demand of 1 . By setting $u_{e}^{*}=1$ for all edges $e$ we obtain a feasible capacity vector and we notice that $u^{*}$ is a vertex $\mathfrak{P}_{\text {sRND. }}$. With respect to $u^{*}$, any set $\{s\} \cup X$ with $X \subset\left\{t_{1}, \ldots, t_{d}\right\}$ defines a binding cut-set inequality. Since there are $2^{d}$ possible choices for $X$, we have $2^{d}$ binding cut-set inequalities at the basic solution $u^{*}$.

\section{Robust Network Design with a Finite Scenario List}

\subsection{A Flow-Based Integer Linear Programming Formulation}

When the uncertainty set $\mathfrak{D}=\left\{b^{1}, \ldots, b^{k}\right\}$ is finite, there is a natural, flow-based integer linear programming formulation of the sRND problem. It contains a set of flow variables for each scenario together with the corresponding flow-conservation and capacity constraints [16]:

$$
\begin{aligned}
& \min \sum_{\{i, j\} \in E} c_{i j} u_{i j} \\
& \text { s.t. } \quad \sum_{\{i, j\} \in E}\left(f_{i j}^{q}-f_{j i}^{q}\right)=b_{i}^{q} \quad \text { for all } i \in V, q=1, \ldots, k \\
& f_{i j}^{q}+f_{j i}^{q} \leq u_{i j} \quad \text { for all }\{i, j\} \in E, q=1, \ldots, k \\
& f_{i j}^{q}, f_{j i}^{q} \geq 0 \quad \text { for all }\{i, j\} \in E, q=1, \ldots, k \\
& u_{i j} \in \mathbb{Z}_{\geq 0} \quad \text { for all }\{i, j\} \in E
\end{aligned}
$$

This formulation is similar to classical integer multicommodity flow (MCF) formulations. The only difference is that in the robust context the $b$-flows $f^{1}, \ldots, f^{k}$ are not simultaneous and thus do not share the edge capacities. Like for the MCF problem, the finite sRND with integral capacities is NP-hard [41], while its fractional variant can be solved in polynomial time (as is proven by the above compact linear programming formulation). Another property is shared with the MCF problem. While the size of the scenario-expanded formulation (IP-F) is polynomial in the input size, it grows impractically large when the number of scenarios (or commodities, in the MCF case) is high. We therefore concentrate on the cut based formulation for the rest of this 
article. We notice, however, that both formulations are equivalent in the sense of the following corollary of Theorem 6. In particular, (IP-CS) can be seen as an orthogonal projection of (IP-F).

Corollary 6. A vector $u \in \mathbb{R}_{>0}^{E}$ is feasible for the linear programming relaxation of the capacity formulation (IP-CS) iff there exist flows $f^{1}, \ldots, f^{k}$ such that $\left(f^{1}, \ldots, f^{k}, u\right)$ is feasible for the linear programming relaxation of the flow formulation (IP-F).

In the non-robust case, the capacity formulation can be obtained by applying Benders' decomposition [12] to the flow formulation, see e.g. [33], and although Benders' original decomposition technique yields a slightly weaker version of (IP-CS), the same principle applies here.

\subsection{Polynomial Time Separation of Cut-Set Inequalities}

In order to use formulation (IP-CS) in practice, we need a fast separation algorithm for its constraints, i.e., we need to decide if a given capacity vector $u^{*}$ violates any cut-set constraints on a network $G=(V, E)$ with uncertainty set $\mathfrak{D}$. We show in this section how this can be achieved when $\mathfrak{D}$ is finite.

To this end, we define an auxiliary graph $\hat{G}=(V \cup\{s\}, \hat{E})$ with

$$
\hat{E}:=E \cup\{(s, \tau) \mid \tau \in V\} .
$$

We now iterate over all scenarios in $\mathfrak{D}$. For some fixed scenario $b \in \mathfrak{D}$, we obtain a cost function for the edges of $\hat{G}$ by extending $u^{*}$ to $\hat{E}$ :

$$
\hat{u}_{e}^{*}:= \begin{cases}-b_{\tau}, & \text { if } e=\{s, \tau\} \\ u_{e}^{*}, & \text { otherwise. }\end{cases}
$$

Then, we can rewrite the value $b(X \cup\{s\})$ of any minimum $s$-cut $X \cup\{s\}$ in $\hat{G}$ as

$$
\operatorname{val}_{b}(X \cup\{s\})=\sum_{e \in \delta_{\hat{G}}(X \cup\{s\})} \hat{u}_{e}^{*}=\sum_{\substack{e \in \delta_{\hat{G}}(X \cup\{s\}) \\ s \notin e}} \hat{u}_{e}^{*}+\sum_{\substack{e \in \delta_{\hat{G}}(X \cup\{s\}) \\ s \in e}} \hat{u}_{e}^{*}=\sum_{e \in \delta_{G}(X)} u_{e}^{*}-\sum_{i \in V \backslash X} b_{i} .
$$

Therefore, any minimum $s$-cut $X \cup\{s\}$ satisfies that $\sum_{i \in V \backslash X} b_{i} \geq 0$ - as otherwise, $(V \backslash X) \cup\{s\}$ has a better objective value. As a consequence, the value of $X \cup\{s\}$ is exactly the slack of the cut-set inequality that $X$ would induce if $b$ was the only scenario. The slack of the true cut-set inequality induced by $X$ can only be smaller and therefore we know that if $\operatorname{val}_{b}(X \cup\{s\})<0$, then also

$$
0>\sum_{e \in \delta_{G}(X)} u_{e}^{*}-\sum_{i \in V \backslash X} b_{i} \geq \sum_{e \in \delta_{G}(X)} u_{e}^{*}-\max _{b \in \mathfrak{D}}\left|\sum_{i \in V \backslash X} b_{i}\right|
$$

and $X$ defines a violated cut-set inequality in $G$. On the other hand, if some $X \subseteq V$ induces a violated cut-set inequality, then there is a scenario $b^{*} \in \mathfrak{D}$ such that

$$
0>\sum_{e \in \delta_{G}(X)} u_{e}^{*}-\max _{b \in \mathfrak{D}}\left|\sum_{i \in V \backslash X} b_{i}\right|=\sum_{e \in \delta_{G}(X)} u_{e}^{*}-\sum_{i \in V \backslash X} b_{i}^{*}=\operatorname{val}_{b^{*}}(X \cup\{s\})
$$


since we can again assume w.l.o.g. that $\sum_{i \in V \backslash X} b_{i}^{*} \geq 0$. Thus, by computing a minimum cut on $\hat{G}$ for each scenario, we can find up to $|\mathfrak{D}|$ violated cut-set inequalities or decide that none exist.

In the construction of $\hat{G}$, the signs of the used edge weights are mixed (i.e., positive and negative). In general, the problem of finding a minimum cut in an arbitrary graph with mixed weights is NP-hard. In our case, however, all edges with negative weight are incident to $s$. This allows us to use a construction for star-negative graphs by McCormick, Rao and Rinaldi [35] which reduces the problem to an ordinary minimum $s$-t-cut problem with non-negative weights. Since this construction changes the size of $G$ by a constant only, we obtain the main theorem of this section.

Theorem 7. Let $(V, E, \mathfrak{D})$ be an instance of the $s R N D$ problem and let $u^{*} \in \mathbb{R}_{>0}^{E}$. Then, we can find a cut-set inequality that is violated by $u^{*}$ or decide that no such inequality exists in time $O\left(|\mathfrak{D}| \cdot T_{\text {mincut }}\right)$, where $T_{\text {mincut }}$ denotes the time need to compute a minimum cut in $G=(V, E)$.

Any maximum flow algorithm can be used to compute a minimum s-t-cut. We implemented the preflow-push algorithm by Goldberg and Tarjan $[25,19]$ with the highest label strategy and the gap heuristic. We stop the algorithm when a maximum preflow is found and thus omit its second stage. This results in an overall runtime of $\Theta\left(|\mathfrak{D}| \cdot|V|^{2} \cdot \sqrt{|E|}\right)$ for the separation procedure.

\subsection{Separating 3-Partition Inequalities more Efficiently}

The assumption that $\mathfrak{D}$ is finite does not only help us to find an efficient separation procedure for cut-set inequalities; it also enables us to find a more efficient alternative to the general 3-partition separation algorithm from Section 2. There, we observed that we can obtain valid 3-partition inequalities by combining two cut-set inequalities with small slack. Instead of enumerating all pairs of binding cut-set inequalities as in Section 2, however, we can now develop an algorithm whose runtime is linear in the number of binding cut-set inequalities.

The key observation for this more efficient algorithm is the following: Our cut-set separation algorithm yields an inequality with maximum violation. Thus, if we try to separate a point $u^{*}$ that already satisfies all cut-set inequalities, it returns an inequality with minimum slack. We use this fact to search for candidates for the zero-half cut generation in our algorithm MinCutZH: For each binding cut-set inequality $\left(\mathrm{CS}_{S}\right)$ in the current LP relaxation, we call the cut-set separation from the previous subsection on the subgraph $G[S]$ that is induced by $S$. This yields up to $|\mathfrak{D}|$ cut-sets $T_{1} \ldots, T_{k} \subset S$. By adding up $\left(\mathrm{CS}_{T_{i}}\right),\left(\mathrm{CS}_{S \backslash T_{i}}\right)$ and $\left(\mathrm{CS}_{T_{i} \cup S \backslash T_{i}}\right)=\left(\mathrm{CS}_{S}\right)$ we thus obtain one 3-partition inequality for each $i=1, \ldots, k$. This algorithm mhas a running time of $O\left(C \cdot|\mathfrak{D}| \cdot T_{\text {mincut }}\right)$ where $C$ is the number of binding cut-set inequalities in the current LP relaxation and $T_{\text {mincut }}$ again denotes the time needed to compute a minimum $s$ - $t$-cut in $G$. It thus depends linearly on the number of binding cut-set inequalities.

Apart from the running time, the algorithm has another advantage over EnumZH: There might be good candidate cut-set inequalities that are not part of the current LP solution - and these can only be found by MinCutZH. On the other hand, we cannot guarantee that the right hand 
side of $\left(\mathrm{CS}_{S}\right)+\left(\mathrm{CS}_{T}\right)+\left(\mathrm{CS}_{S}\right)$ is odd and therefore it can happen that MinCutZH does not find a violated 3-partition inequality even though one exists (as is the case with EnumZH).

\section{Robust Network Design with Polyhedral Demand Uncertainties}

Duffield et al. [22] propagate the Hose demand polytope for multi-commodity network design. Rather than specifying demands for all pairs of nodes (which can be impractical in large networks), they propose to define two bounds for each node $i$ that limit how much flow in total the node $i$ can send to (or receive from, respectively) all other nodes. This is a natural model as these bounds can stem from technical specifications, legal contracts or educated guesses by experienced engineers.

Pesenti, Rinaldi and Ukovich [39] propose a similar model for single-commodity flows: They start from the multi-commodity model and limit the traffic demand $r_{i j}$ for each pair of nodes by an individual upper and lower bound, $r_{i j}^{\max }$ and $r_{i j}^{\min }$. Given any such matrix $r=\left(r_{i j}\right)_{i, j \in V}$ with $r_{i j}^{\min } \leq r_{i j} \leq r_{i j}^{\max }$, they aggregate the commodities to a demand vector $\left(b_{i}\right)_{i \in V}:=\left(\sum_{j \in V} r_{i j}-\right.$ $\left.r_{j i}\right)_{i \in V}$. Any demand vector that can be obtained in this fashion is a scenario that needs to be considered in the optimization. This problem is called the Network Containment Problem in the literature. Pesenti, Rinaldi and Ukovich subsequently propose to solve the problem with a branch-and-cut algorithm based on a cut-set formulation and a separation MIP.

We propose a different adaptation of the Hose model that is simpler and does not have a point-to-point traffic component. For each node $i \in V$, we define an upper bound $b_{i}^{\max }$ and a lower bound $b_{i}^{\text {min }}$. We then say that any supply- and demand vector that obeys these bounds while remaining balanced is a possible scenario for our optimization. The resulting uncertainty set is the polytope

$$
\mathfrak{H}\left(V, b^{\text {min }}, b^{\text {max }}\right):=\left\{b \in R^{V} \mid b_{i} \in\left[b_{i}^{\text {min }}, b_{i}^{\text {max }}\right] \text { for all } i \in V \text { and } \sum_{i \in V} b_{i}=0\right\} .
$$

Due to its similarity to the Hose uncertainty set that is used for multi-commodity network design problems, we call it the single commodity Hose polytope. In the following, we assume that our uncertainty set $\mathfrak{D}$ is the polytope $\mathfrak{H}\left(V, b^{\min }, b^{\max }\right)$ and denote the corresponding (sRND) problem by (sRND-Hose).

\subsection{Complexity of Robust Network Design with Single Commodity Hose Demands}

Finding an optimum integer solution for (sRND-Hose) is NP-hard, as the problem contains Steiner Tree as a special case (see [41] for a similar reduction for finite $\mathfrak{D}$ ).

Theorem 8. The (sRND-Hose) problem is NP-hard.

Proof. Let $I=\left(V_{I}, E_{I}, c_{I}, \mathfrak{T}\right)$ be an input for the Steiner Tree problem, i.e., suppose that $G_{I}=$ $\left(V_{I}, E_{I}\right)$ is an undirected graph with edge weights $c_{I}$ and that $\emptyset \subsetneq \mathfrak{T} \subseteq V_{I}$ is a set of terminals that 
need to be connected at minimum cost. Steiner Tree is NP-hard [29]. Then, finding an optimum solution for $I$ is equivalent to finding an optimum solution for the following sRND instance $J$ : Select some arbitrary node $s \in \mathfrak{T}$. We set $\hat{b}_{s}^{\min }=0$ and $\hat{b}_{s}^{\max }=1$. For all other nodes $i \in \mathfrak{T} \backslash\{s\}$, set $\hat{b}_{i}^{\min }=-1$ and $\hat{b}_{i}^{\max }=0$. Now, the vertices of $\mathfrak{H}\left(V_{I}, \hat{b}^{\text {min }}, \hat{b}^{\text {max }}\right)$ are exactly the scenarios $b$ where $b_{s}=1$ and $b_{i}=-1$ for some node $i \in \mathfrak{T}$. This means that in any feasible solution for $J$, there must be a path of capacity 1 from $s$ to all terminals $i \in \mathfrak{T} \backslash\{s\}$. Also, if the support of any feasible integer solution for $J$ contains a cycle, then one edge of the cycle can be deleted. Thus, any optimum solution for $J$ induces a Steiner Tree and any Steiner Tree solution for $I$ defines a solution for $J$; moreover, the costs of the solutions are identical in both cases. Thus, when $\mathfrak{D}=\mathfrak{H}\left(V, b^{\min }, b^{\max }\right)$, solving sRND is at least as hard as solving Steiner Tree.

We shall see in the remainder of the section that the separation problem for cut-set inequalities is also NP-hard for (sRND-Hose). This proves that (sRND-Hose) remains hard even if we relax the integrality requirement.

\subsection{Separating Cut Set Inequalities over $\mathfrak{H}\left(V, b^{\min }, b^{\max }\right)$}

Finding optimum solutions for the sRND problem in practice becomes significantly harder when the uncertainty set is the polytope $\mathfrak{H}\left(V, b^{\min }, b^{\text {max }}\right)$. Following our previous approach, we want to to solve the linear programming relaxation of the capacity-based formulation (IP-CS) in order to generate dual bounds in a branch-and-bound algorithm. As opposed to the case that $\mathfrak{D}$ is finite, however, finding a cut-set inequality with maximum violation will turn out to be NP-hard when $\mathfrak{D}=\mathfrak{H}\left(V, b^{\min }, b^{\max }\right)$. The NP-hardness of this problem is somewhat surprising: We could expect to solve the separation problem for (IP-CS) with a minimum cut algorithm. Here, however, the main obstacle is to compute the correct right hand side for a given cut $S$ inside of the minimum cut computation. When $\mathfrak{D}$ is finite, we can simply enumerate all possible scenarios $b$ and interpret $b$ as linear node costs that are easily integrated into any minimum cut algorithm. When $\mathfrak{D}=\mathfrak{H}\left(V, b^{\min }, b^{\max }\right)$, however, this is no longer possible, as a more sophisticated optimization problem needs to be solved to obtain the correct right hand side for the cut-set inequalities. This is true even though computing the correct right-hand side for a fixed $S$ is possible in polynomial time; the difficulty lies in computing it while computing a minimum cut.

Summarizing, our problem is that (IP-CS) contains a non-trivial optimization problem on the right hand side. Still, solving such formulations is at the core of robust optimization and several ideas from the literature can be applied here. We observe, for instance, that if we interpret the $b_{i}$ on the right hand side of (IP-CS) as variables, we obtain a bi-level optimization problem. It minimizes the capacities on the outer level and maximizes the total demands on the its inner level, i.e., the right hand side of each of the cut-set inequalities. Now, if the right hand side optimization problem was a minimization problem, we could collapse (IP-CS) a single level hoping to obtain inequalities that can be separated more easily. Thus, we only need to replace the linear program $\max _{b \in D}\left|\sum_{i \in S}\right|$ by its dual. This technique has been applied successfully to the multi-commodity robust network design problem by Ben-Ameur and Kerivin [10] in the case 
of static routing. In their case, it results in a separable linear formulation. Applying the same technique to the multi-commodity robust network design problem with dynamic routing leads to a non-convex quadratic separation problem, as was shown by Mattia [34]. The same is true in our case. However, when the underlying network flow has several commodities, Mattia observes that linearizing the separation problem yields a mixed integer linear program with big- $M$ constraints. Ben-Tal and Nemirovski [11] give a general solution algorithm for robust linear programs, requiring only that the uncertainty set is compact and that separation over it is possible. They show that any linear program with row-wise uncertainty of this type can be optimized by solving an auxilliary linear program for each row of its deterministic (i.e., non-robust) counterpart. Potentially, each auxilliary problem yields a valid cutting plane for the robust formulation. While we can certainly separate over $\mathfrak{H}\left(V, b^{\min }, b^{\max }\right)$, the deterministic counterpart of (IP-CS) unfortunately has an exponential number of rows. We would therefore require an oracle that gives us a row for which the auxilliary problem yields a valid cutting plane. Finding such an oracle is equivalent to solving our original separation problem. Another alternative could be to use a polynomially sized flow-based formulation as deterministic counterpart, but short of introducing a full set of flow-variables for each vertex of $\mathfrak{H}\left(V, b^{\min }, b^{\max }\right)$, it is not clear how to robustify the flow-conservation equalities of such a formulation. We conclude that we need to find an alternative to these standard-techniques if we want to solve our separation problem.

Our first step to a practical separation algorithm is to actually write down the separation problem: The following bi-level program will give us a separating hyperplane for any $u^{*} \notin$ $\mathfrak{P}_{\mathrm{sRND}}(G, \mathfrak{H})$. Since $S \subseteq V$ is variable here, the formulation is not a linear or quadratic program in the strict sense. It can, however, be transformed into a bi-level quadratic program. For now, we stick to the more abstract formulation to benefit from the easier notation. Solving

$$
\min _{S \subseteq V} \sum_{e \in \delta(S)} u_{e}^{*}-\max _{b \in \mathfrak{H}} \sum_{i \in S} b_{i}
$$

yields a cut-set inequality that is violated if and only if the optimum objective value of (H-SEP) is negative. As in the finite case, we do not need to take the absolute value of the second sum, as we can assume w.l.o.g. that the total balance $\sum_{i \in S} b_{i}$ is non-negative in an optimum solution $(S, b)$. Moreover, we say that $S$ is a hose source set iff $\sum_{i \in S} b_{i}^{\max } \geq 0$ and $\sum_{i \in V \backslash S} b_{i}^{\min } \leq 0$. We only consider hose source sets in the following. If $S$ is not a hose source set, then either $\mathfrak{H}\left(V, b^{\min }, b^{\max }\right)$ is empty or $\sum_{i \in S} b_{i}<0$ for all $b \in \mathfrak{H}\left(V, b^{\min }, b^{\max }\right)$. Finally, we say that a hose source set $S$ is limiting, if $\sum_{i \in S} b_{i}^{\max } \leq-\sum_{i \in V \backslash S} b_{i}^{\min }$. Otherwise, we say that $V \backslash S$ is limiting. We will show next that we can re-write the inner level

$$
B_{S}:=\max _{b \in \mathfrak{H}} \sum_{i \in S} b_{i} \quad \text { for a fixed } S \subseteq V
$$

such that (H-SEP) reduces to a single-level mixed integer linear program.

We proceed in two steps: First, we give an algorithm that both functions as a scenario separation and proves that there exists a solution of a certain value for (MAX-B). At the same time, we will see that we can compute the value of the solution with a closed formula without 
actually running the algorithm. This will enable us to integrate the solution value into (H-SEP). Secondly, we prove that our solution maximizes (MAX-B).

To get a better intuition for the algorithm, suppose that $0 \in \mathfrak{H}\left(V, b^{\min }, b^{\max }\right)$ and consider the following preliminary method to find an optimum solution for (MAX-B). We start with the vector $b \equiv 0 \in \mathfrak{H}\left(V, b^{\min }, b^{\max }\right)$ and our aim is to install as much supply as possible in $S$. Equivalently, we could try to install as much demand as possible in $S$, but since we assumed w.l.o.g. that the maximum total balance of $S$ is non-negative, we rather stick to the maximum supply case. We now select an arbitrary node $i \in S$ with $b_{i}<b_{i}^{\max }$ and another arbitrary node $j \in V \backslash S$ with $b_{j}>b_{j}^{\min }$. If no such nodes can be found, the algorithm stops. Finally, we increase $b_{i}$ by one unit and, at the same time, decrease $b_{j}$ by one unit to maintain a balanced vector.

To analyse the algorithm, we observe that it maintains $\sum_{i \in S} b \leq \sum_{i \in S} b_{i}^{\max }$ and $\sum_{i \in S} b_{i}=$ $-\sum_{i \in V \backslash S} b_{i} \leq-\sum_{i \in V \backslash S} b_{i}^{m i n}$. The algorithm stops as soon as equality holds in one of the conditions. Thus, if $b$ is the vector that we obtain once the algorithm stops, we have $\sum_{i \in S} b_{i}=$ $\min \left\{\sum_{i \in S} b_{i}^{\max },-\sum_{i \in V \backslash S} b_{i}^{\min }\right\}$ and we realize that we can compute the value of this solution without actually running the algorithm. Also, increasing the objective value of $b$ further would make $b$ necessarily imbalanced.

The idea of our preliminary algorithm was to start from a feasible vector and to then increase its objective value. We follow the same idea in the case that $0 \in\left[b_{i}^{\min }, b_{i}^{\max }\right]$ for all $i \in V$, however, we need a slightly more involved algorithm to do so. The problem is that the starting vector $b \equiv 0$ might be infeasible. More verbosely, the node bounds can force us to install supply on a node in $V \backslash S$ or to install demand on a node in $S$ and thereby change the amount of imbalance that we have to distribute. The bounds can also force us to install a minimum amount of supply or demand on some nodes in $S$ or $V \backslash S$ - which is a problem if we already distributed all the imbalance before reaching such nodes. Both problems can be solved by starting from a different vector. This is why, in contrast to the preliminary algorithm, we start with a vector $b$ that simply satisfies $b_{i} \in\left[b_{i}^{\min }, b_{i}^{\max }\right]$ for all $i \in V$ and then make sure that $\sum_{i \in V} b_{i}=0$ in a second phase.

Additionally, the runnning time of our previous algorithm is only pseudopolynomial, as the algorithm needs $\min \left\{\sum_{i \in S} b_{i}^{\max }, \sum_{i \in V \backslash S} b_{i}^{\min }\right\}$ many iterations. We overcome this second problem by increasing the $b$ values by as much possible in every iteration. To know this amount, it is necessary to precompute which of the two bounds is reached first, i.e., whether $S$ or $V \backslash S$ is the limiting set. If $S$ has more limiting bounds than $V \backslash S$, we set $b_{i}=b_{i}^{\max }$ for all $i \in S$; otherwise, we set $b_{i}=b_{i}^{\min }$ for all $i \in V \backslash S$. In both cases, it only remains to distribute the inbalance of $b$ among the nodes in the non-limiting set. To do this, we iterate over all nodes $i$ in the non-limiting set in arbitrary order and decrease or increase $b_{i}$ as much as possible in the first and second case, respectively. See Algorithm 1 for the pseudo-code of this procedure. When the algorithm stops with a balanced vector $b$, we obtain again a solution $b$ of value $\min \left\{\sum_{i \in S} b_{i}^{\max },-\sum_{i \in V \backslash S} b_{i}^{\min }\right\}$.

Lemma 9. Given a hose source set $\emptyset \subsetneq S \subsetneq V$, Algorithm 1 computes a scenario $b \in \mathfrak{H}\left(V, b^{\text {min }}, b^{\text {max }}\right)$ with

$$
\sum_{i \in S} b_{i}=\min \left\{\sum_{i \in S} b_{i}^{\max },-\sum_{i \in V \backslash S} b_{i}^{\min }\right\}
$$


or it correctly decides that $\mathfrak{H}\left(V, b^{\text {min }}, b^{\text {max }}\right)$ is empty.

Proof. Let $L=S$ or $L=V \backslash S$ be the set that limits how much supply we can install in $S$. We prove the correctness of the algorithm by showing that Lines 13-27 maintain two invariants: (1) At all times, $b$ respects all bounds, i.e., $b_{i} \in\left[b_{i}^{\min }, b_{i}^{\max }\right]$ for all $i \in V$. (2) At all times, $r$ stores the balance of our current $b$ vector, i.e. $r=\sum_{i \in V} b_{i}$.

We establish Invariant 1 in lines 3-9 and 10/11 for $i \in L$ and $i \in F$, respectively. Line 12 establishes Invariant 2. Suppose now that $r<0$ in line 13 (the other case works analogeously). We already know that both invariants hold before the first iteration of the loop in lines 14-19 and we assume by induction that the same is true before the $j$-th iteration, for some $j \geq 2$. Suppose that the $j$-th iteration considers $i \in F$. Then, $b_{i}$ is at most increased to $b_{i}+b_{i}^{\max }-b_{i}=b_{i}^{\max }$, i.e. Invariant 1 is maintained. Also, $r$ is changed by the same value as $b_{i}$ and thus still stores the current balance of $b$. This means that Invariant 2 still holds. When the algorithm stops with $r=0$, we have found a scenario $b \in \mathfrak{H}\left(V, b^{\min }, b^{\max }\right)$. Also, by our choice in lines 3-9, we have $\sum_{i \in S} b_{i}=\sum_{i \in S} b_{i}^{\max }$ if $S$ is limiting and $\sum_{i \in S} b_{i}=-\sum_{i \in V \backslash S}=-\sum_{i \in V \backslash S} b_{i}^{\min }$ otherwise. If the algorithm stops with $r<0$, then $m=b_{i}^{\max }-b_{i}$ in all iterations and thus, $b_{i}=b_{i}^{\max }$ for all $i \in F$ where $b_{i}^{\max }>0$. From line 11 , we know that $b_{i}=b_{i}^{\max }$ for all $i \in F$ with $b_{i}^{\max }<0$ and our initialization guarantees $0=b_{i}=b_{i}^{\max }$ for all the $i \in F$ with $b_{i}^{\max }=0$. We conclude that $0>r=\sum_{i \in F} b_{i}=\sum_{i \in F} b_{i}^{\max }$. If $F=S$, we directly have a contradiction to $S$ being a hose source set. If $F=V \backslash S$ instead, we also have $b_{i}=b_{i}^{\max }$ for all $i \in L$. It follows that $\sum_{i \in V} b_{i}=\sum_{i \in V} b_{i}^{\max }<$ 0 . Now, let $b^{\prime} \in \mathfrak{H}\left(V, b^{\min }, b^{\text {max }}\right)$. Then, $\sum_{i \in V} b_{i}^{\prime} \leq \sum_{i \in V} b_{i}^{\max }<0$ which is a contradiction to $\sum_{i \in V} b_{i}^{\prime}=0$. Consequently, $\mathfrak{H}\left(V, b^{\min }, b^{\max }\right)=\emptyset$.

It remains to show that Algorithm 1 computes an optimum scenario for (MAX-B).

Theorem 10. Let $S \subseteq V$ be a hose source set. Then

$$
B_{S}=\min \left\{\sum_{i \in S} b_{i}^{\max },-\sum_{i \in V \backslash S} b_{i}^{\min }\right\} .
$$

Proof. For $i=1, \ldots,|V|$, introduce dual variables $v_{i}, \lambda_{i}$ for the upper/lower bound constraints of $b_{i}$, repectively, and define a dual variable $\beta$ for the balance constraint. This gives us the dual (MAX-B*) of (MAX-B)

$$
\begin{aligned}
\min \sum_{i \in V} b_{i}^{\max } v_{i}-\sum_{i \in V} b_{i}^{\min } \lambda_{i} & \\
v_{i}-\lambda_{i}+\beta \geq 1 & \text { for all } i \in S \\
v_{i}-\lambda_{i}+\beta \geq 0 & \text { for all } i \in V \backslash S \\
v_{i}, \lambda_{i} \geq 0 & \text { for all } i \in V
\end{aligned}
$$

If $\sum_{i \in S} b_{i}^{\text {max }} \leq-\sum_{i \in V \backslash S} b_{i}^{\text {min }}$, running Algorithm 1 gives us a scenario $b$ with $\sum_{i \in S} b_{i}=\sum_{i \in S} b_{i}^{\text {max }}$. We choose $v_{i}=1$ for all $i \in S, v_{i}=0$ for all $i \in V \backslash S, \lambda_{i}=0$ for all $i \in V$ and finally $\beta=0$. 
Our choice $(v, \lambda, \beta)$ is feasible for $\left(H_{S}^{*}\right)$ and satisfies complementary slackness with $b$. Otherwise, we suppose $\sum_{i \in S} b_{i}^{\max }>-\sum_{i \in V \backslash S} b_{i}^{\min }$ and Algorithm 1 yields a scenario $b$ with $\sum_{i \in S}=$ $-\sum_{i \in V \backslash S} b_{i}^{\min }$. Choosing $\lambda_{i}=1$ for all $i \in V \backslash S, \lambda_{i}=0$ for all $i \in S, v_{i}=0$ for all $i \in V$ and $\beta=1$ is a feasible solution for $\left(H_{S}^{*}\right)$ and $b$ satisfies complementary slackness with $(v, \lambda, \beta)$.

Theorem 10 tells us that we can write (H-SEP) as the much easier problem

$$
\min _{S \subseteq V} \sum_{e \in \delta(S)} u_{e}^{*}-\min \left\{\sum_{i \in S} b_{i}^{\max },-\sum_{i \in V \backslash S} b_{i}^{\min }\right\} .
$$

In this formulation, we omitted the constraint that $\sum_{i \in S} b_{i} \geq 0$, because the inner part of the problem yields a negative value if it is violated. Thus, omitting the constraint does not produce more optimum solutions. We can now write (H-SEP') as a MIP that is a maximum cut problem with additional constraints:

$$
\begin{array}{rlrl}
\min \sum_{\{i, j\} \in E} u_{i j}^{*} y_{i j}-B & \\
B \leq \sum_{i \in V} x_{i} b_{i}^{\text {max }} & \\
B \leq-\sum_{i \in V}\left(1-x_{i}\right) b_{i}^{\text {min }} & \\
x_{i}-x_{j} \leq y_{i j} & & \\
x_{j}-x_{i} \leq y_{i j} & \text { for all }\{i, j\} \in E \\
x_{i} & \in\{0,1\} & \text { for all }\{i, j\} \in E \\
y_{i j} & \in\{0,1\} & \text { for all } i \in V \\
& \text { for all }\{i, j\} \in E
\end{array}
$$

(IP-H-SEP)

The MIP will not give us an actual worst-case scenario; however, we can easily call Algorithm 1 on the set $S:=\left\{i \in V \mid x_{i}=1\right\}$ to obtain one. If we want more than one worst-case scenario, we can even call it several times while permuting the order in which it considers the nodes.

In contrast to the finite case, separating cut-set inequalities in the polyhedral case is NP-hard, as we show in the following theorem by a reduction from minimum expansion. Chekuri, Oriolo, Scutellà and Shepherd [18] show the same result for the multi-commodity case. In fact, they also use a (more complicated) reduction from minimum expansion.

Theorem 11. Given an instance $\left(G, \mathfrak{H}\left(V, b^{\min }, b^{\max }\right)\right)$ of the (sRND-Hose) problem and a fractional capacity vector $u \in \mathbb{R}^{E}$, the problem of finding a cut-set inequality that is violated by $u$ is $N P$-hard. In particular, the feasibility test for u is co-NP-complete.

Proof. Minimum expansion is defined in the following way: Given an undirected graph $G=$ $(V, E)$ and edge capacities $u_{e}$ for all edges $e \in E$, find a set $\emptyset \subsetneq S \subsetneq V$ with $|S| \leq|V| / 2$ that minimizes the expansion $\sum_{e \in \delta(S)} u_{e} /|S|$ of $G$. Minimum expansion is NP-hard [30, Section 3.2]. 
If we have an input $(V, E, u)$ for minimum expansion, we can define an instance for the cut-set separation problem on the same graph $G=(V, E)$. Set $\hat{b}_{i}^{\max }:=1$ and $\hat{b}_{i}^{\min }=-1$ for all $i \in V$. We claim that $G$ has an expansion of strictly less than 1 if and only if there is a violated cut-set inequality with respect to $u$ and $\mathfrak{H}\left(V, \hat{b}^{\min }, \hat{b}^{\max }\right)$.

By our definition of $\hat{b}^{\max }$ and $\hat{b}^{\min }$, we obtain from Theorem 10 that $B_{S}=|S|$ for any $S \subseteq V$ with $|S| \leq|V| / 2$. Thus, there is a violated cut-set inequality in $G$ iff for some $S, \sum_{e \in \delta(S)} u_{e}<B_{S}=|S|$. This is equivalent to $\sum_{e \in \delta(S)} u_{e} /|S|<1$.

\section{Computational results}

In this section we describe the outcome of our extensive computational campaign conducted to assert the effectiveness of the cut-set formulation within a classical branch-and-cut framework for both the finite and Hose cases. The branch-and-cut algorithm is implemented in C++ within the ABACUS 3.2U2 framework [28] and run on an Intel XEON $54102.3 \mathrm{GHz}$ with $3 \mathrm{~GB}$ RAM, and Cplex 12.1 is used as an LP solver inside our branch and cut. The main ingredients to enhance the basic scheme are described in the following, while Section 5.1 and Section 5.2 report the results on the finite and Hose cases, respectively.

Preprocessing. We partition the graph into its biconnected components as suggested in [16]. It is straight-forward to generalize the approach to the polytopal case.

Cutting Plane Separation. We use the cut-set separation both for the finite and the Hose case, as described in Sections 3 and 4, respectively. In the Hose case, after the exact separation from Section 4 is invoked, we repeatedly call Algorithm 1 to obtain a list of 10 non-routable scenarios. Then, the polynomial separation of Section 3 is called until the 10 scenarios can be routed. The separation algorithm EnumZH is called at the root node only, as well as the $\left\{0, \frac{1}{2}\right\}$-cut separation code by Andreello, Caprara and Fischetti [5] which is called at the root node when the other algorithms fail.

Primal Heuristics. We execute several fast primal heuristics at each branch-and-cut node when no more cut-set inequality is violated. They are all based on rounding operations. More precisely,

- The Simple rounding rounds up all fractional values to produce a feasible solution.

- The Cycle rounding looks for a cycle $C$ with only fractional edges by a depth first search. On $C$ the heuristic rounds down the edge with the smallest fractional value, say, $p$ and increases the capacity of all other edges on $C$ by $p$. When no more cycles are found, all remaining fractional capacities are rounded up. 
- The Shortest Path rounding works in the same way as the Cycle rounding, but it obtains the cycle $C$ by removing an edge with a smallest fractional value and connecting its endnodes by a shortest path of fractional edges.

In the finite case, we also use the rounding heuristic described in [16]. Finally, we use the large neighborhood search heuristic introduced in [3,4], but only at the root node, in the finite case and with a time limit of 120 CPU seconds.

Settings. Very few special settings have been used within the branch-and-cut framework provided by ABACUS. Namely, we used aggressive strong branching, the branch-and-cut tree is traversed in best-first order, and we removed non-binding cutting planes after 10 iterations.

\subsection{Experiments with Finite Uncertainty Sets}

Testbed. We consider four different classes of instances for our experiments. (These instances, as well as those for the Hose case (Section 5.2), are available upon request from the authors.) Each instance consists of a network topology and a scenario set.

- BLS: The instances have been used in [16] and are based on realistic network topologies introduced in [2].

- JMP: The instances are generated according to the method in [27] with zero-one balances as proposed in [4].

- SNDLib: The SNDLib [38] is an established standard benchmark set for real-world network topologies. We augmented the real-world topologies with random balances to adapt the instances to our specific problems.

- PA: The preferential attachment model [8] defines a standard way to create realistic networks: For some parameter $a \geq 2$, one starts with a complete graph on $a$ nodes and iteratively adds more nodes and edges to the network. When a new node $v$ is inserted, it connects to exactly $a$ existing nodes. In this way, the parameter $a$ controls the density of the graph. The probability that $v$ connects to an existing node $w$ is proportional to the degree of $w$. Again, we augmented the resulting network topologies with random balances.

Comparison with the flow formulation. Our experiments for the finite case compare the cutset formulation (IP-CS) with the flow formulation introduced in [16]. For the BLS instances the comparison is performed with the algorithm in [16] that solved the flow formulation by enhancing it through target cuts (see, Section 1). We have access to the original computational data by [16] and conducted the experiments on the same machines, making direct comparison possible. For the other set of instances, instead, the flow formulation has been solved as a blackbox MIP through Cplex 12.1 by using default settings and in single-thread mode. This is to provide a fair comparison with the sequential ABACUS implementation. Finally, the time limit for each instance was set to 4 hours of CPU time. 
Description of the tables. In Tables 1-4 we show instances that could be solved to optimality by both of the compared methods and averages over sub-classes of instances for each table entry. Computing times are expressed in CPU seconds. We first show the instance size and the percentage gap between the optimum fractional and integer solution values. Recall that the flow formulation and the cut-set one are proven to be equivalent in terms of LP relaxation bound. For each method we show the number of instances that could be solved to optimality within $14,400 \mathrm{CPU}$ seconds (4 hours) and in brackets number of instances that stopped due to memory limit of 3 GB. Then, we report the average CPU time over all instances that could be solved to optimality by both methods and the corresponding number of branch-and-bound nodes. The root gap reported is the average percentage gap of the dual root bound (after all cuts were added) with respect to the optimum integer solution value. Finally, we report the time that is needed to solve the LP-relaxation. For the cut-set formulation only, we also report the overall separation time and the overall heuristic time. For the PA instances (Table 4) the results for each size are average over $a \in\{2,3,4,5,6,7\}$.

Results. Table 1 shows that our branch-and-cut algorithm based on the cut-set formulation is superior to the branch-and-cut algorithm (also ABACUS-based) in [16] both in terms of the number of solved instances and the CPU time. In particular, these instances turn out to be rather easy for our algorithm that only has some issues due to memory limits. Specifically, the memory limit prevents us from finding the optimum solution of 10 out of 1,156 instances.

Instances JMP (Table 2) turn out to be much more challenging and the comparison with the flow formulation solved by Cplex is interesting. Until $|V|=35$ both methods can solve all instances (in roughly the same computing time) and we can observe that the cut-set formulation amended by $\left\{0, \frac{1}{2}\right\}$-cuts gives a better bound than the flow formulation with Cplex cuts. On larger instances $|V| \geq 40$ both algorithms start to suffer and the algorithm based on the cut-set formulation frequently reaches the memory limit. Instead, when Cplex is unable to solve the problem it is because of the time limit (14,400 CPU seconds), which is a clear indication that the formulation became too big. As the bound at the root node is better for our algorithm, this behavior seems to indicate that the memory limit reached by our algorithm is likely a software limit (essentially due to the less sophisticated implementation of ABACUS with respect to Cplex) and not a problem of the formulation, whose LP size is always kept under control through cut purging.

The above analysis is confirmed by the results on Tables 3 and 4 for the classes SNDlib and PA, respectively. Especially on the PA instances one can start too appreciate that, for large values of $|V|$ and many scenarios, the cut-set formulation becomes more effective while the flow formulation is too large. That can be expected as the separation limits the size of the cut-set LP to the needed cuts. For Tables 3 and 4 the numbers in brackets for the "\#solved(m)" column of the flow formulation refer to the number of times the memory limit is reached. So, it is worth mentioning that for PA instances, cplex reaches both the time and the memory limit (the number of solved instances plus those not solved due to the memory limit is sometimes smaller 
than 180), showing that the size of the flow formulation gives rise to all sort of issues. The two numbers instead almost always sum to 180 in the cut-set formulation case, thus confirming that the management of the tree of ABACUS is likely to be the issue. Nevertheless, for large instances with many scenarios our algorithm can solve many more instances in significantly shorter computing times.

\subsection{Experiments with the Hose Uncertainty Set}

In order to obtain a flow formulation in the Hose uncertainty case, we would have to convert the linear description of the Hose polytope $\mathfrak{H}\left(V, b^{\min }, b^{\max }\right)$ into a list of its vertices. This can be done with a software like PORTA [20]. Table 5 shows that this approach is not practical: Already for small instances, we cannot rely on being able to convert the description within 1800 seconds and, additionally, the list of vertices can easily become too large to be useful. Therefore, we cannot present a comparison with the flow formulation in the Hose case.

Testbed. To limit the space needed to present our results, we only report the results on the most general instances, i.e., the SNDLib and PA topologies. The Hose uncertainty sets have been generated according to three different distributions:

- geometric: The width of the demand intervals is chosen with a geometric distribution, i.e., there are many nodes with narrow demand intervals and few nodes with broad intervals. The center of the intervals is chosen uniformly at random.

- uniform: Both the width and the center of the intervals are chosen uniformly at random for each node.

- zero-one: All intervals are $[-1,1]$.

Description of the tables. In Tables 6 and 7, we report the CPU time and number of solved instances for the random Hose instances, grouped by network topology in the SNDLib case and according to the density parameter $a$ in the PA case, respectively. We also show the number of times that the separation MIP needs to be solved on average over all separation calls. Again, we use a time limit of 4 hours.

Results. The results in Table 6 show that the branch-and-cut algorithm based on the flow formulation is effective in the Hose uncertainty case. More precisely, very few of the SNDlib instances cannot be solved to optimality and both the computing times and the number of branchand-bound nodes are small on average. The same holds for the PA instances (Table 7) where the difficulty grows with the value of $a$. In terms of the difference of the random distribution, the behavior on geometric and uniform instances is quite similar, while the zero-one case turns 
out to be rather easy, except for 9 instances with $|V|=100$ and $a=6$ and one instance with $|V|=100$ and $a=7$.

In Table 8 we report a disaggregated picture of our cut-set based algorithm. We consider the PA instances for the three distributions and $a=6$. In addition to the previous information, we show the time ("ip-sep-time") needed to solve the exact separation MIP (see Section 4), and the corresponding number of calls, "ip sepcalls (in \%)". The results for other values of parameter $a$ are comparable. The disaggregated results in Table 8 allow us to assert that the quality of both the LP and root lower bounds is very high. However, the difficulty of the instances with respect to the finite uncertainty case seems to be associated with closing the small gap within the time limit. Indeed, all unsolved PA instances reach the time limit (not a memory limit). This is due to the size of the resulting problems: The LPs start to be time consuming as well as the separation time, especially due to exact separation MIP. 


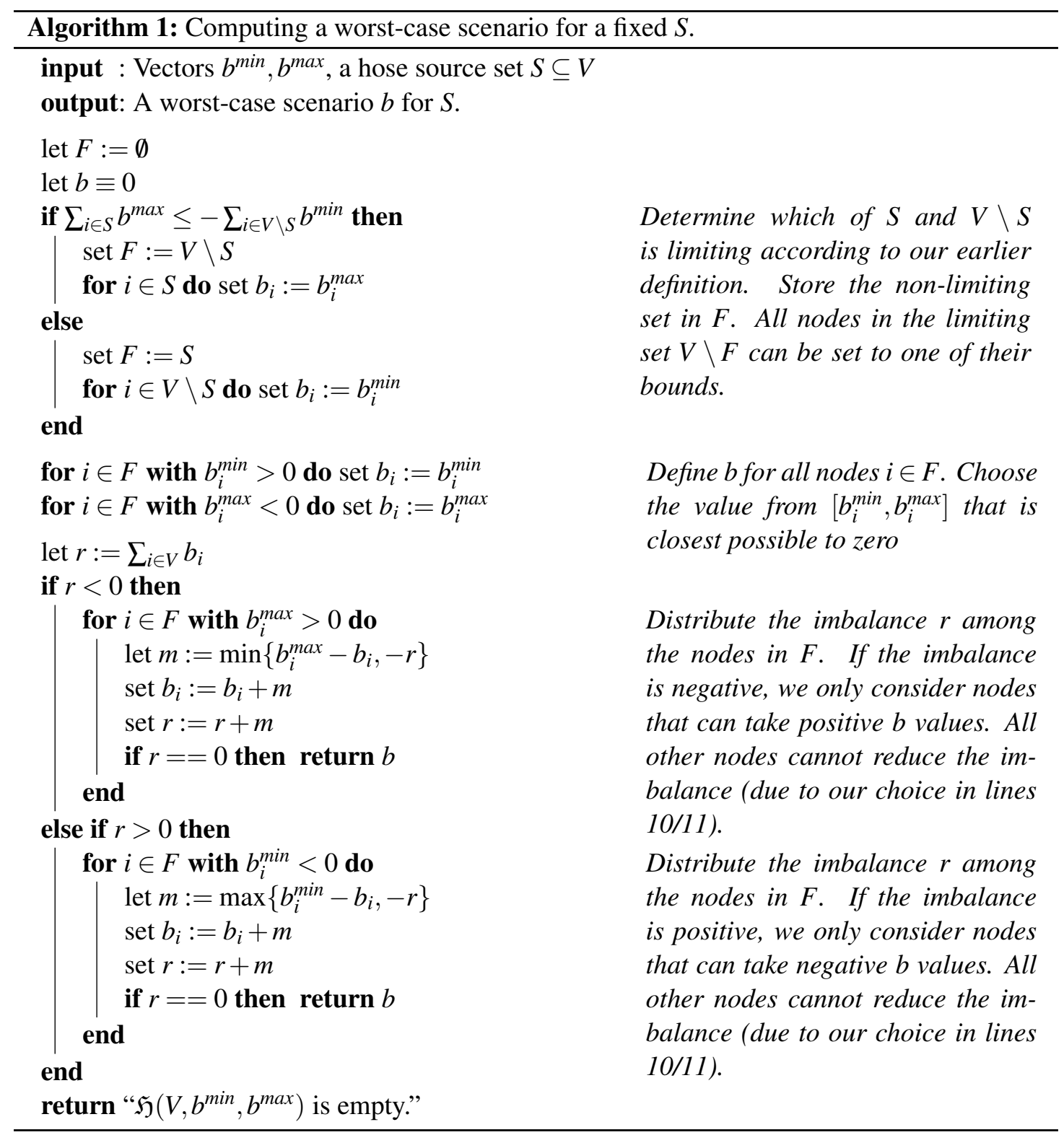




\begin{tabular}{|c|c|c|c|c|c|c|c|c|c|c|c|c|c|}
\hline \multirow{2}{*}{\multicolumn{2}{|c|}{$\bar{z}$}} & \multirow[b]{2}{*}{$\underline{A}$} & \multirow[b]{2}{*}{$\begin{array}{l}\text { 泀 } \\
\text { 茾 }\end{array}$} & \multirow[b]{2}{*}{ 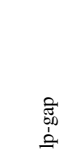 } & \multicolumn{7}{|c|}{ Cut-Set formulation (CS) } & \multicolumn{2}{|c|}{ BLS [16] } \\
\hline & & & & & $\begin{array}{l}\widehat{\Xi} \\
\vec{D} \\
\stackrel{\partial}{0} \\
\# \\
\#\end{array}$ & $\begin{array}{l}\stackrel{\mathscr{E}}{\mathrm{E}} \\
\text { 它 }\end{array}$ & 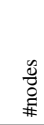 & $\begin{array}{l}\text { 今. } \\
\stackrel{0}{0} \\
\stackrel{0}{0} \\
\stackrel{0}{0}\end{array}$ & 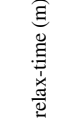 & 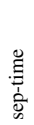 & 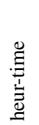 & $\begin{array}{l}\widehat{\Xi} \\
\vec{D} \\
\frac{\partial}{0} \\
0 \\
\#\end{array}$ & 嗀 \\
\hline $0 \leq|V|$ & $\leq 149$ & 2 & 153 & $0.02 \%$ & $153(0)$ & 2 & 111 & $0.00 \%$ & $0(0)$ & 0 & 0 & 153 & 0.7 \\
\hline $0 \leq|V|$ & $\leq 149$ & 3 & 153 & $0.03 \%$ & $152(1)$ & 7 & 265 & $0.00 \%$ & $0(0)$ & 0 & 0 & 152 & 1.1 \\
\hline $0 \leq|V|$ & $\leq 149$ & 4 & 153 & $0.03 \%$ & $151(2)$ & 2 & 105 & $0.00 \%$ & $0(0)$ & 0 & 0 & 150 & 4.8 \\
\hline $0 \leq|V|$ & $\leq 149$ & 5 & 185 & $0.02 \%$ & $182(3)$ & 0 & 127 & $0.00 \%$ & $0(0)$ & 0 & 0 & 183 & 5.9 \\
\hline $150 \leq|V|$ & $\leq 299$ & 2 & 68 & $0.00 \%$ & $67(1)$ & 2 & 78 & $0.00 \%$ & $0(0)$ & 1 & 0 & 66 & 85.6 \\
\hline $150 \leq|V|$ & $\leq 299$ & 3 & 68 & $0.01 \%$ & $68(0)$ & 45 & 205 & $0.00 \%$ & $0(0)$ & 8 & 0 & 61 & 4.9 \\
\hline $150 \leq|V|$ & $\leq 299$ & 4 & 68 & $0.00 \%$ & $66(2)$ & 2 & 95 & $0.00 \%$ & $0(0)$ & 1 & 0 & 63 & 27.3 \\
\hline $150 \leq|V|$ & $\leq 299$ & 5 & 68 & $0.00 \%$ & $67(1)$ & 82 & 186 & $0.00 \%$ & $0(0)$ & 11 & 0 & 62 & 141.2 \\
\hline $300 \leq|V|$ & $\leq 499$ & 2 & 60 & $0.00 \%$ & $60(0)$ & 0 & 197 & $0.00 \%$ & $0(0)$ & 0 & 0 & 60 & 81.3 \\
\hline $300 \leq|V|$ & $\leq 499$ & 3 & 60 & $0.00 \%$ & $60(0)$ & 0 & 169 & $0.00 \%$ & $0(0)$ & 0 & 0 & 60 & 103.4 \\
\hline $300 \leq|V|$ & $\leq 499$ & 4 & 60 & $0.00 \%$ & $60(0)$ & 0 & 221 & $0.00 \%$ & $0(0)$ & 0 & 0 & 59 & 129.8 \\
\hline $300 \leq|V|$ & $\leq 499$ & 5 & 60 & $0.00 \%$ & $60(0)$ & 0 & 547 & $0.00 \%$ & $0(0)$ & 0 & 0 & 55 & 166.8 \\
\hline
\end{tabular}

Table 1: Comparison to [16] on the BLS class. We use the same grouping and the same machines as the original authors.

\begin{tabular}{|c|c|c|c|c|c|c|c|c|c|c|c|c|c|c|c|}
\hline \multirow[b]{2}{*}{$\Sigma$} & \multirow[b]{2}{*}{ 포 } & \multirow[b]{2}{*}{$\underline{\overline{\mathrm{A}}}$} & \multirow[b]{2}{*}{$\begin{array}{l}\stackrel{0}{\circ} \\
. \\
\stackrel{0}{\circ} \\
\stackrel{0}{\circ}\end{array}$} & \multicolumn{7}{|c|}{ Cut-Set formulation (CS) } & \multicolumn{5}{|c|}{ Flow formulation (CPLEX) } \\
\hline & & & & 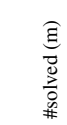 & 思 & $\begin{array}{l}\text { vo } \\
0\end{array}$ & 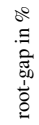 & 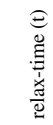 & 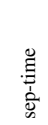 & 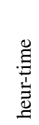 & 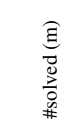 & $\begin{array}{l}\text { 咅 } \\
\text { 号 }\end{array}$ & $\begin{array}{l}\frac{\mathscr{s}}{0} \\
\frac{0}{\#}\end{array}$ & $\begin{array}{l}\circ \\
. \\
. \\
\text { 今. } \\
\stackrel{0}{0} \\
\stackrel{0}{0}\end{array}$ & 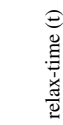 \\
\hline 25 & 104 & 5 & 13.3 & $3(0)$ & 1 & 46 & 2.9 & $0(0)$ & 0 & 0 & $3(0)$ & 0 & 410 & 7.7 & $0(0)$ \\
\hline 25 & 104 & 10 & 17.1 & $3(0)$ & 24 & 2016 & 7.1 & $0(0)$ & 3 & 0 & $3(0)$ & 26 & 2701 & 12.2 & $0(0)$ \\
\hline 30 & 121 & 5 & 10.6 & $3(0)$ & 7 & 436 & 2.5 & $0(0)$ & 1 & 0 & $3(0)$ & 5 & 1175 & 5.6 & $0(0)$ \\
\hline 30 & 121 & 10 & 14.3 & $3(0)$ & 125 & 6875 & 6.6 & $0(0)$ & 15 & 1 & $3(0)$ & 123 & 12661 & 9.5 & $0(0)$ \\
\hline 35 & 155 & 5 & 12.3 & $3(0)$ & 75 & 6157 & 5.3 & $0(0)$ & 7 & 0 & $3(0)$ & 9 & 1808 & 7.1 & $0(0)$ \\
\hline 35 & 155 & 10 & 12.3 & $3(0)$ & 1196 & 47858 & 6.2 & $0(0)$ & 115 & 20 & $3(0)$ & 597 & 31355 & 9.2 & $0(0)$ \\
\hline 40 & 182 & 5 & 17.2 & 2 ( 1 ) & 51 & 1886 & 6.8 & $0(0)$ & 8 & 0 & $3(0)$ & 6 & 1121 & 12.0 & $0(0)$ \\
\hline 40 & 182 & 10 & - & $0(3)$ & - & - & - & $0(0)$ & - & - & $3(0)$ & - & - & - & $0(0)$ \\
\hline 45 & 223 & 5 & 16.1 & $1(2)$ & 15 & 243 & 5.6 & $0(0)$ & 6 & 0 & $3(0)$ & 10 & 1106 & 8.4 & $0(0)$ \\
\hline 45 & 223 & 10 & - & 0 ( 3$)$ & - & - & - & $0(0)$ & - & - & $1(0)$ & - & - & - & $0(0)$ \\
\hline 50 & 254 & 5 & - & 0 ( 3 ) & - & - & - & $0(0)$ & - & - & $2(0)$ & - & - & - & $0(0)$ \\
\hline 50 & 274 & 10 & - & 0 ( 3$)$ & - & - & - & $0(0)$ & - & - & $0(0)$ & - & - & - & $0(0)$ \\
\hline
\end{tabular}

Table 2: Computational results for the instances of the JMP class. We consider 3 instances for each pair $(|V|,|\mathfrak{D}|)$. 


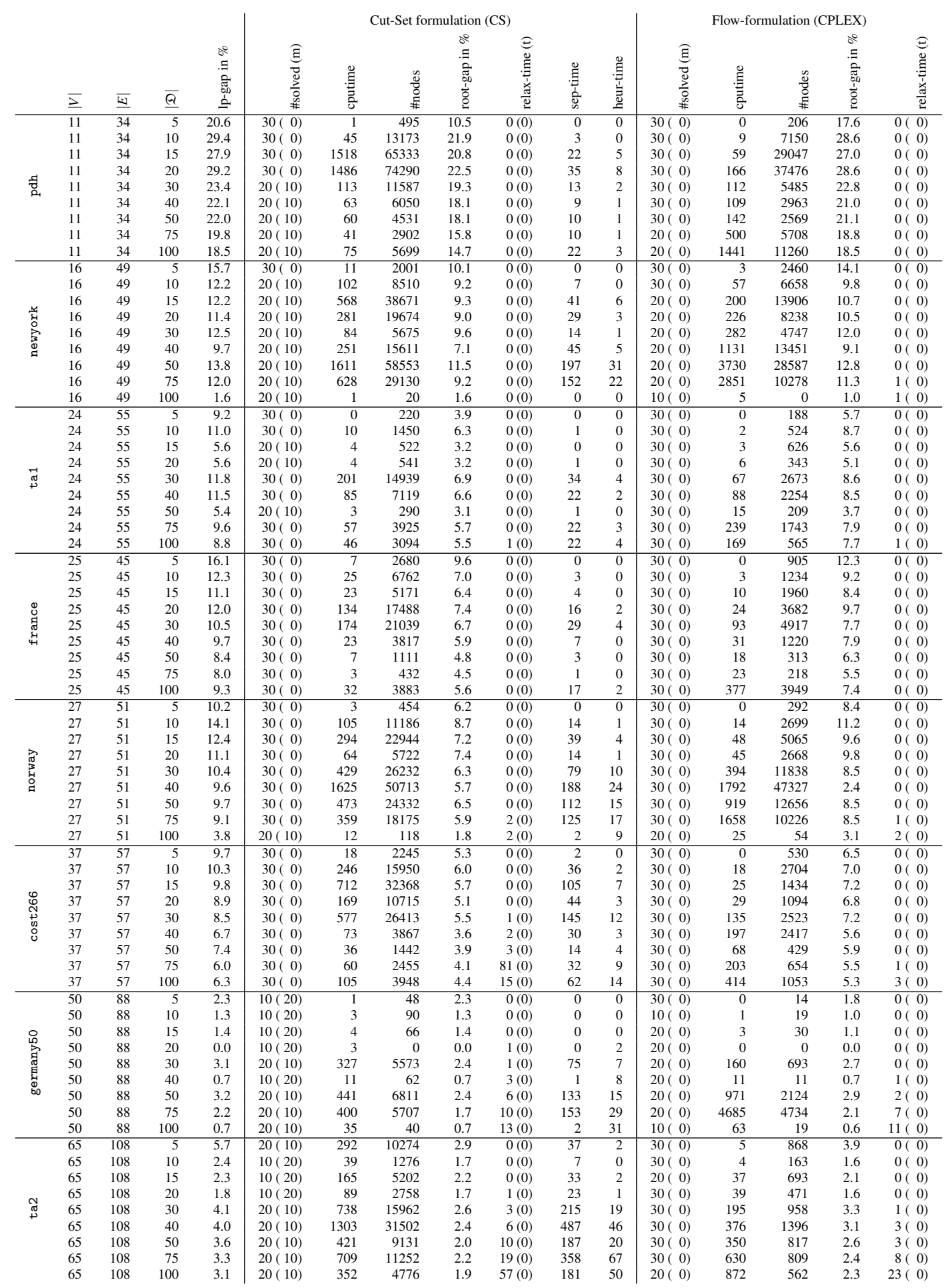

Table 3: Computational results for the instances of the SNDlib class. We consider 30 instances for each network topology and for each number of scenarios $|\mathfrak{D}| \in$ $\{5,10,15,20,30,40,50,75,100\}$. 


\begin{tabular}{|c|c|c|c|c|c|c|c|c|c|c|c|c|c|c|c|}
\hline \multirow[b]{2}{*}{$\geq$} & \multirow[b]{2}{*}{ 퍼 } & \multirow[b]{2}{*}{$\underline{\bar{A}}$} & \multirow[b]{2}{*}{$\begin{array}{l}\circ \\
\cong \\
0 \\
0.0 \\
\vdots \\
=\end{array}$} & & & -Set forr & lation & & & & & Flow fo & ulation & PLEX & \\
\hline & & & & 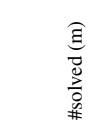 & 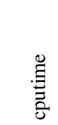 & 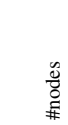 & 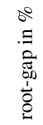 & 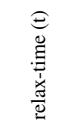 & 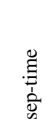 & 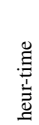 & $\begin{array}{l}\widehat{\mathrm{g}} \\
\widetilde{\Xi} \\
\frac{\partial}{0} \\
0 \\
\#\end{array}$ & $\begin{array}{l}\text { 音 } \\
\text { 它 }\end{array}$ & 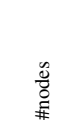 & 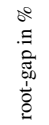 & 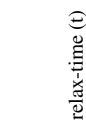 \\
\hline 20 & 76 & 5 & 8.1 & $180(0)$ & 0 & 21 & 2.5 & $0(0)$ & 0 & 0 & $180(0)$ & 0 & 43 & 4.6 & $0(0)$ \\
\hline 20 & 76 & 10 & 8.6 & $180(0)$ & 0 & 51 & 3.2 & $0(0)$ & 0 & 0 & $180(0)$ & 0 & 101 & 5.5 & $0(0)$ \\
\hline 20 & 76 & 30 & 7.8 & $180(0)$ & 0 & 45 & 3.0 & $0(0)$ & 0 & 0 & $180(0)$ & 3 & 102 & 5.0 & $0(0)$ \\
\hline 20 & 76 & 50 & 7.3 & $180(0)$ & 0 & 42 & 2.7 & $0(0)$ & 0 & 0 & $180(0)$ & 7 & 88 & 4.8 & $0(0)$ \\
\hline 20 & 76 & 75 & 6.8 & $180(0)$ & 0 & 31 & 2.4 & $0(0)$ & 0 & 0 & $180(0)$ & 15 & 80 & 4.4 & $0(0)$ \\
\hline 20 & 76 & 100 & 6.3 & $180(0)$ & 0 & 25 & 2.0 & $0(0)$ & 0 & 0 & $180(0)$ & 19 & 63 & 4.0 & $1(0)$ \\
\hline 25 & 98 & 5 & 9.3 & $180(0)$ & 1 & 163 & 3.7 & $0(0)$ & 0 & 0 & $180(0)$ & 0 & 258 & 6.0 & $0(0)$ \\
\hline 25 & 98 & 10 & 9.7 & $180(0)$ & 5 & 489 & 4.4 & $0(0)$ & 1 & 0 & $180(0)$ & 7 & 651 & 6.8 & $0(0)$ \\
\hline 25 & 98 & 30 & 8.4 & $180(0)$ & 6 & 366 & 3.9 & $0(0)$ & 2 & 0 & $180(0)$ & 57 & 684 & 6.2 & $0(0)$ \\
\hline 25 & 98 & 50 & 8.0 & $180(0)$ & 7 & 323 & 3.7 & $0(0)$ & 3 & 0 & $180(0)$ & 140 & 681 & 6.0 & $1(0)$ \\
\hline 25 & 98 & 75 & 7.7 & $180(0)$ & 9 & 312 & 3.6 & $0(0)$ & 4 & 0 & $180(0)$ & 306 & 693 & 5.8 & $2(0)$ \\
\hline 25 & 98 & 100 & 7.4 & $180(0)$ & 10 & 319 & 3.4 & $0(0)$ & 6 & 0 & $180(0)$ & 497 & 689 & 5.6 & $4(0)$ \\
\hline 30 & 121 & 5 & 6.3 & $180(0)$ & 3 & 213 & 2.5 & $0(0)$ & 0 & 0 & $180(0)$ & 1 & 212 & 4.0 & $0(0)$ \\
\hline 30 & 121 & 10 & 6.6 & $180(0)$ & 9 & 529 & 2.8 & $0(0)$ & 2 & 0 & $180(0)$ & 15 & 469 & 4.5 & $0(0)$ \\
\hline 30 & 121 & 30 & 5.9 & $180(0)$ & 8 & 322 & 2.6 & $0(0)$ & 3 & 0 & $180(0)$ & 122 & 469 & 4.1 & $0(0)$ \\
\hline 30 & 121 & 50 & 5.4 & $180(0)$ & 12 & 365 & 2.4 & $0(0)$ & 5 & 0 & $180(0)$ & 399 & 541 & 3.9 & $2(0)$ \\
\hline 30 & 121 & 75 & 5.2 & $180(0)$ & 10 & 233 & 2.4 & $0(0)$ & 5 & 0 & $178(0)$ & 544 & 397 & 3.8 & $5(0)$ \\
\hline 30 & 121 & 100 & 4.9 & $180(0)$ & 9 & 178 & 2.3 & $0(0)$ & 5 & 0 & $172(0)$ & 792 & 368 & 3.6 & $8(0)$ \\
\hline 35 & 143 & 5 & 7.7 & $180(0)$ & 8 & 399 & 3.2 & $0(0)$ & 1 & 0 & $180(0)$ & 2 & 326 & 4.9 & $0(0)$ \\
\hline 35 & 143 & 10 & 8.2 & $180(0)$ & 36 & 1610 & 3.9 & $0(0)$ & 6 & 0 & $180(0)$ & 39 & 967 & 5.7 & $0(0)$ \\
\hline 35 & 143 & 30 & 7.6 & $180(0)$ & 79 & 2609 & 3.8 & $0(0)$ & 21 & 2 & $180(0)$ & 582 & 1950 & 5.7 & $1(0)$ \\
\hline 35 & 143 & 50 & 7.1 & $180(0)$ & 57 & 1539 & 3.6 & $0(0)$ & 21 & 2 & $180(0)$ & 1327 & 1447 & 5.3 & $4(0)$ \\
\hline 35 & 143 & 75 & 6.6 & $180(0)$ & 45 & 998 & 3.3 & $0(0)$ & 21 & 2 & $174(0)$ & 2540 & 1216 & 5.0 & $10(0)$ \\
\hline 35 & 143 & 100 & 5.6 & $180(0)$ & 30 & 488 & 2.8 & $0(0)$ & 16 & 2 & $158(0)$ & 2906 & 729 & 4.2 & $14(0)$ \\
\hline 40 & 166 & 5 & 6.6 & $180(0)$ & 6 & 259 & 2.4 & $0(0)$ & 1 & 0 & $180(0)$ & 2 & 283 & 3.8 & $0(0)$ \\
\hline 40 & 166 & 10 & 6.7 & $180(0)$ & 15 & 568 & 2.8 & $0(0)$ & 3 & 0 & $180(0)$ & 24 & 510 & 4.2 & $0(0)$ \\
\hline 40 & 166 & 30 & 6.0 & $180(0)$ & 28 & 772 & 2.5 & $0(0)$ & 9 & 0 & $180(0)$ & 338 & 788 & 4.0 & $2(0)$ \\
\hline 40 & 166 & 50 & 5.5 & $180(0)$ & 26 & 582 & 2.4 & $0(0)$ & 11 & 1 & $179(0)$ & 944 & 742 & 3.8 & $6(0)$ \\
\hline 40 & 166 & 75 & 4.9 & $180(0)$ & 18 & 357 & 2.1 & $0(0)$ & 10 & 1 & $169(0)$ & 1496 & 550 & 3.4 & $13(0)$ \\
\hline 40 & 166 & 100 & 4.4 & $180(0)$ & 11 & 187 & 1.8 & $0(0)$ & 7 & 0 & $132(30)$ & 1405 & 387 & 3.1 & $21(0)$ \\
\hline 45 & 188 & 5 & 5.8 & $180(0)$ & 6 & 226 & 2.1 & $0(0)$ & 2 & 0 & $180(0)$ & 1 & 235 & 3.2 & $0(0)$ \\
\hline 45 & 188 & 10 & 6.2 & $180(0)$ & 15 & 576 & 2.6 & $0(0)$ & 5 & 0 & $180(0)$ & 27 & 518 & 4.0 & $0(0)$ \\
\hline 45 & 188 & 30 & 5.2 & $180(0)$ & 21 & 553 & 2.3 & $0(0)$ & 9 & 0 & $180(0)$ & 318 & 645 & 3.6 & $2(0)$ \\
\hline 45 & 188 & 50 & 4.8 & $180(0)$ & 23 & 477 & 2.2 & $0(0)$ & 13 & 0 & $180(0)$ & 999 & 629 & 3.4 & $9(0)$ \\
\hline 45 & 188 & 75 & 4.3 & $180(0)$ & 20 & 322 & 2.0 & $0(0)$ & 13 & 1 & $174(0)$ & 1985 & 572 & 3.2 & $21(0)$ \\
\hline 45 & 188 & 100 & 4.2 & $180(0)$ & 21 & 374 & 1.9 & $0(0)$ & 13 & 1 & $89(90)$ & 1883 & 645 & 3.0 & $26(30)$ \\
\hline 50 & 211 & 5 & 6.9 & $173(7)$ & 113 & 3042 & 2.9 & $0(0)$ & 14 & 0 & $180(0)$ & 17 & 909 & 4.0 & $0(0)$ \\
\hline 50 & 211 & 10 & 6.2 & $152(28)$ & 196 & 4766 & 2.8 & $0(0)$ & 29 & 2 & $180(0)$ & 102 & 1372 & 4.0 & $0(0)$ \\
\hline 50 & 211 & 30 & 5.2 & 146 ( 34$)$ & 192 & 3900 & 2.4 & $0(0)$ & 52 & 5 & $146(0)$ & 913 & 1743 & 3.5 & $5(0)$ \\
\hline 50 & 211 & 50 & 4.7 & 143 ( 37$)$ & 167 & 3083 & 2.2 & $0(0)$ & 61 & 6 & $133(0)$ & 1664 & 1721 & 3.2 & $18(0)$ \\
\hline 50 & 211 & 75 & 4.4 & $153(27)$ & 92 & 1672 & 2.0 & $0(0)$ & 46 & 5 & 117 (29) & 2101 & 1064 & 3.0 & $38(0)$ \\
\hline 50 & 211 & 100 & 4.7 & $152(28)$ & 58 & 1099 & 2.5 & $0(0)$ & 33 & 3 & $45(120)$ & 1356 & 888 & 3.1 & $23(60)$ \\
\hline 60 & 256 & 5 & 6.3 & 169 (11) & 165 & 3272 & 2.7 & $0(0)$ & 20 & 1 & $180(0)$ & 20 & 842 & 3.7 & $0(0)$ \\
\hline 60 & 256 & 10 & 5.3 & $133(47)$ & 269 & 5525 & 2.4 & $0(0)$ & 43 & 4 & $177(0)$ & 125 & 1407 & 3.3 & $0(0)$ \\
\hline 60 & 256 & 30 & 4.5 & $135(45)$ & 279 & 4841 & 2.2 & $0(0)$ & 79 & 9 & $142(0)$ & 1290 & 1884 & 3.0 & $12(0)$ \\
\hline 60 & 256 & 50 & 3.9 & $134(46)$ & 154 & 2409 & 1.9 & $0(0)$ & 61 & 6 & $119(0)$ & 2523 & 1458 & 2.6 & $40(0)$ \\
\hline 60 & 256 & 75 & 3.8 & $138(42)$ & 103 & 1623 & 1.8 & $0(0)$ & 52 & 5 & $68(90)$ & 2112 & 1188 & 2.6 & $56(30)$ \\
\hline 60 & 256 & 100 & 4.0 & 141 ( 39$)$ & 24 & 477 & 1.5 & $0(0)$ & 17 & 1 & $30(150)$ & 382 & 509 & 2.3 & $169(60)$ \\
\hline 70 & 301 & 5 & 4.5 & $135(45)$ & 181 & 2909 & 1.9 & $0(0)$ & 23 & 1 & $180(0)$ & 21 & 694 & 2.5 & $0(0)$ \\
\hline 70 & 301 & 10 & 3.5 & $109(71)$ & 228 & 3921 & 1.5 & $0(0)$ & 40 & 3 & $160(0)$ & 92 & 919 & 2.2 & $1(0)$ \\
\hline 70 & 301 & 30 & 3.0 & 107 ( 73 ) & 212 & 2979 & 1.3 & $0(0)$ & 61 & 5 & $120(0)$ & 821 & 1042 & 1.9 & $18(0)$ \\
\hline 70 & 301 & 50 & 2.6 & $110(70)$ & 219 & 2399 & 1.3 & $0(0)$ & 77 & 7 & $111(0)$ & 2278 & 867 & 1.8 & $80(0)$ \\
\hline 70 & 301 & 75 & 3.3 & $110(70)$ & 279 & 3899 & 1.4 & $0(0)$ & 138 & 13 & $44(120)$ & 1431 & 1111 & 2.2 & $121(60)$ \\
\hline 70 & 301 & 100 & - & $107(72)$ & - & - & - & $0(0)$ & - & - & $0(180)$ & - & - & - & $129(120)$ \\
\hline 80 & 346 & 5 & 3.3 & $107(73)$ & $\begin{array}{l}169 \\
\end{array}$ & 2260 & 1.2 & $0(0)$ & 21 & 1 & $180(0)$ & 10 & 405 & 1.8 & $0(0)$ \\
\hline 80 & 346 & 10 & 2.6 & $91(89)$ & 148 & 2649 & 1.1 & $0(0)$ & 28 & 1 & $145(0)$ & 48 & 440 & 1.5 & $2(0)$ \\
\hline 80 & 346 & 30 & 2.5 & $93(87)$ & 368 & 3951 & 1.3 & $0(0)$ & 95 & 7 & $100(0)$ & 1215 & 765 & 1.6 & $39(0)$ \\
\hline 80 & 346 & 50 & 3.2 & $93(85)$ & 366 & 3652 & 1.5 & $0(0)$ & 131 & 12 & $66(60)$ & 2402 & 781 & 2.0 & $131(0)$ \\
\hline 80 & 346 & 75 & 3.6 & $89(87)$ & 102 & 1527 & 1.3 & $0(0)$ & 64 & 4 & $30(150)$ & 512 & 634 & 2.0 & $304(60)$ \\
\hline 80 & 346 & 100 & - & $88(90)$ & - & - & - & $0(0)$ & - & - & $0(180)$ & - & - & - & $29(150)$ \\
\hline 90 & 391 & 5 & 2.7 & $88(92)$ & 330 & 4393 & 1.1 & $0(0)$ & 41 & 2 & $177(0)$ & 11 & 381 & 1.4 & $0(0)$ \\
\hline 90 & 391 & 10 & 1.2 & 68 (111) & 136 & 2142 & 0.6 & $0(0)$ & 30 & 1 & $121(0)$ & 31 & 223 & 0.7 & $3(0)$ \\
\hline 90 & 391 & 30 & 1.0 & $67(111)$ & 261 & 1603 & 0.7 & $0(0)$ & 55 & 3 & $85(0)$ & 2070 & 640 & 0.8 & $66(0)$ \\
\hline 90 & 391 & 50 & 1.6 & $71(109)$ & 634 & 7437 & 0.9 & $0(0)$ & 262 & 21 & $49(90)$ & 1544 & 1814 & 1.1 & $141(30)$ \\
\hline 90 & 391 & 75 & 2.1 & $72(108)$ & 1285 & 11281 & 1.2 & $0(0)$ & 586 & 47 & $23(150)$ & 1767 & 1426 & 1.4 & 220 (120) \\
\hline 90 & 391 & 100 & - & $72(107)$ & - & - & - & - & - & - & $0(180)$ & - & - & - & - \\
\hline 100 & 436 & 5 & 2.0 & $81(99)$ & 186 & 2042 & 0.7 & $0(0)$ & 25 & 0 & $170(0)$ & 6 & 230 & 1.0 & $0(0)$ \\
\hline 100 & 436 & 10 & 1.3 & $71(109)$ & 264 & 3055 & 0.7 & $0(0)$ & 49 & 2 & $103(0)$ & 104 & 382 & 0.8 & $4(0)$ \\
\hline 100 & 436 & 30 & 1.2 & $67(108)$ & 383 & 3043 & 0.7 & $1(0)$ & 100 & 7 & $63(0)$ & 1753 & 774 & 0.9 & $104(0)$ \\
\hline 100 & 436 & 50 & 1.7 & $66(108)$ & 817 & 6624 & 1.0 & $1(0)$ & 311 & 23 & $38(90)$ & 2010 & 1425 & 1.2 & $221(30)$ \\
\hline 100 & 436 & 75 & - & $62(107)$ & - & - & - & $0(0)$ & - & - & $0(180)$ & - & - & - & $20(150)$ \\
\hline 100 & 436 & 100 & - & $59(110)$ & - & - & - & - & - & - & $0(180)$ & - & - & - & - \\
\hline
\end{tabular}

Table 4: Computational results for the PA class. We report aggregated results over all values of $a \in\{2,3,4,5,6,7\}$, thus having 180 instances for each pair $(|V|,|\mathfrak{D}|)$. 

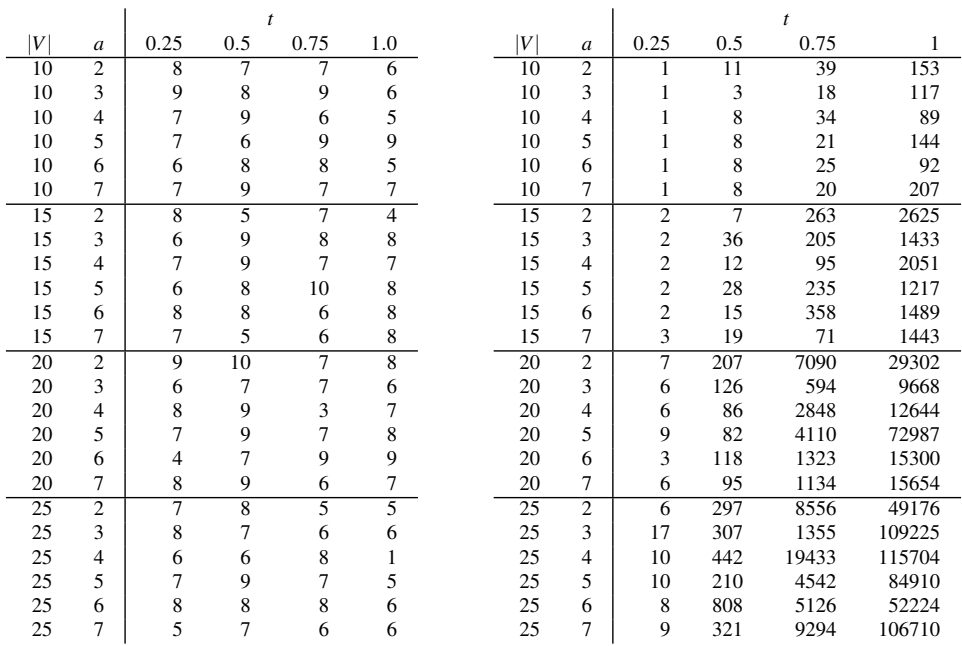

Table 5: Using PORTA to convert the linear description of the instances from the PA Hose class, geometric demand distribution. Grouping by the percentage $t \in\{0.25,0.5,0.75,1.0\}$ of terminal nodes. On the left: Number of instances that could be converted within 1800 seconds. On the right: Resulting average number of vertices of the demand polytope.

\begin{tabular}{|c|c|c|c|c|c|c|c|c|c|c|c|}
\hline & \multirow[b]{2}{*}{ 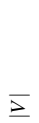 } & \multirow[b]{2}{*}{$\underline{\underline{1}}$} & \multicolumn{3}{|c|}{ geometric } & \multicolumn{3}{|c|}{ uniform } & \multicolumn{3}{|c|}{ zero-one } \\
\hline & & & $\begin{array}{l}\vec{D} \\
\frac{\partial}{D} \\
\#\end{array}$ & 兽 & $\begin{array}{l}\frac{\tilde{v}}{0} \\
\frac{0}{\#}\end{array}$ & $\begin{array}{l}\vec{D} \\
\stackrel{D}{0} \\
\#\end{array}$ & 壱 & $\begin{array}{l}\frac{\tilde{v}}{0} \\
\frac{0}{\#}\end{array}$ & $\begin{array}{l}\vec{D} \\
\text { Dे } \\
0 \\
\# \#\end{array}$ & 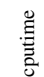 & $\begin{array}{l}\frac{\hat{y}}{0} \\
0 \\
\text { En }\end{array}$ \\
\hline $\mathrm{pdh}$ & 11 & 34 & 39 & 5 & 30 & 40 & 0 & 33 & 40 & 1 & 45 \\
\hline newyork & 16 & 49 & 40 & 19 & 58 & 38 & 61 & 59 & 40 & 0 & 98 \\
\hline ta1 & 24 & 55 & 40 & 154 & 74 & 39 & 331 & 73 & 40 & 0 & 70 \\
\hline france & 25 & 45 & 31 & 13 & 63 & 30 & 38 & 64 & 40 & 0 & 54 \\
\hline norway & 27 & 51 & 38 & 189 & 109 & 39 & 34 & 114 & 40 & 0 & 84 \\
\hline cost 266 & 37 & 57 & 38 & 15 & 183 & 37 & 423 & 217 & 40 & 7 & 203 \\
\hline germany 50 & 50 & 88 & 31 & 411 & 498 & 30 & 239 & 662 & 40 & 172 & 575 \\
\hline ta2 & 65 & 108 & 39 & 558 & 525 & 39 & 38 & 510 & 40 & 0 & 413 \\
\hline
\end{tabular}

Table 6: Computational results on the SNDLib Hose instances. For each of the three distributions, we consider 40 different Hose uncertainty sets per topology. 


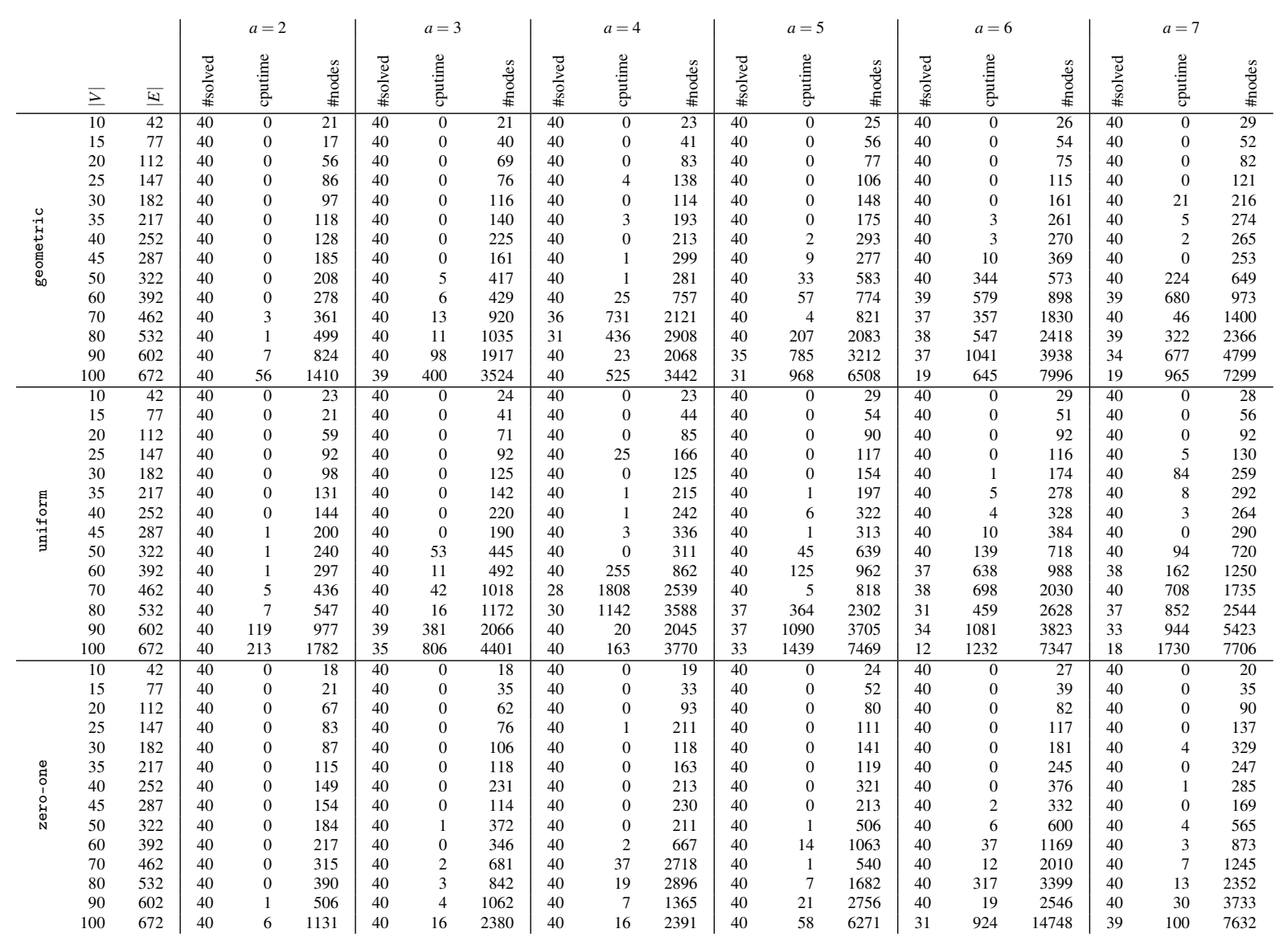

Table 7: Computational results on the PA Hose instances. For each of the three distributions, we consider 40 instances per pair $(|V|,|E|)$ and per $a \in\{2,3,4,5,6,7\}$. 


\begin{tabular}{|c|c|c|c|c|c|c|c|c|c|c|c|c|}
\hline & $\bar{z}$ & $\underline{\underline{\underline{v}}}$ & 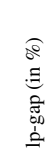 & 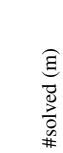 & $\begin{array}{l}\stackrel{\mathscr{\Xi}}{\Xi} \\
\text { 苾 }\end{array}$ & $\begin{array}{l}\frac{n}{0} \\
0\end{array}$ & 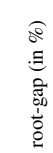 & 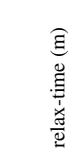 & 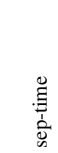 & 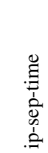 & 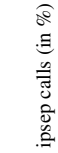 & 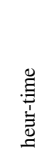 \\
\hline \multirow{14}{*}{ 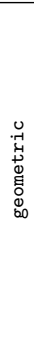 } & 10 & 39 & 0.32 & $40(0)$ & 0 & 25 & 0.00 & $0(0)$ & 0 & 0 & 14.07 & 0 \\
\hline & 15 & 69 & 0.24 & $40(0)$ & 0 & 53 & 0.03 & $0(0)$ & 0 & 0 & 10.45 & 0 \\
\hline & 20 & 99 & 0.17 & $40(0)$ & 0 & 74 & 0.03 & $0(0)$ & 0 & 0 & 10.42 & 0 \\
\hline & 25 & 129 & 0.22 & $40(0)$ & 0 & 114 & 0.03 & $0(0)$ & 0 & 0 & 9.33 & 0 \\
\hline & 30 & 159 & 0.08 & $40(0)$ & 0 & 160 & 0.02 & $0(0)$ & 0 & 0 & 10.27 & 0 \\
\hline & 35 & 189 & 0.16 & $40(0)$ & 3 & 260 & 0.08 & $0(0)$ & 2 & 2 & 12.60 & 0 \\
\hline & 40 & 219 & 0.10 & $40(0)$ & 3 & 269 & 0.04 & $0(0)$ & 3 & 2 & 9.92 & 0 \\
\hline & 45 & 249 & 0.19 & $40(0)$ & 10 & 368 & 0.09 & $0(0)$ & 8 & 6 & 13.10 & 0 \\
\hline & 50 & 279 & 0.15 & $40(0)$ & 344 & 572 & 0.10 & $2(0)$ & 300 & 284 & 16.88 & 0 \\
\hline & 60 & 339 & 0.13 & $39(0)$ & 579 & 897 & 0.11 & $6(0)$ & 517 & 486 & 16.26 & 0 \\
\hline & 70 & 399 & 0.12 & $37(0)$ & 357 & 1829 & 0.09 & $15(0)$ & 330 & 303 & 8.31 & 0 \\
\hline & 80 & 459 & 0.08 & $38(0)$ & 547 & 2417 & 0.06 & $26(0)$ & 481 & 440 & 11.93 & 0 \\
\hline & 90 & 519 & 0.13 & $37(0)$ & 1041 & 3937 & 0.10 & $43(0)$ & 897 & 751 & 8.74 & 0 \\
\hline & 100 & 579 & 0.07 & $19(0)$ & 645 & 7995 & 0.06 & $219(0)$ & 519 & 373 & 3.93 & 0 \\
\hline \multirow{14}{*}{ 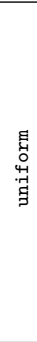 } & 10 & 39 & 0.28 & $40(0)$ & 0 & 28 & 0.01 & $0(0)$ & 0 & 0 & 14.68 & 0 \\
\hline & 15 & 69 & 0.31 & $40(0)$ & 0 & 50 & 0.05 & $0(0)$ & 0 & 0 & 13.50 & 0 \\
\hline & 20 & 99 & 0.32 & $40(0)$ & 0 & 91 & 0.06 & $0(0)$ & 0 & 0 & 11.70 & 0 \\
\hline & 25 & 129 & 0.16 & $40(0)$ & 0 & 115 & 0.05 & $0(0)$ & 0 & 0 & 11.59 & 0 \\
\hline & 30 & 159 & 0.37 & $40(0)$ & 1 & 173 & 0.08 & $0(0)$ & 0 & 0 & 13.47 & 0 \\
\hline & 35 & 189 & 0.20 & $40(0)$ & 5 & 277 & 0.11 & $0(0)$ & 4 & 3 & 14.83 & 0 \\
\hline & 40 & 219 & 0.19 & $40(0)$ & 4 & 327 & 0.08 & $0(0)$ & 3 & 2 & 13.90 & 0 \\
\hline & 45 & 249 & 0.11 & $40(0)$ & 10 & 383 & 0.07 & $1(0)$ & 8 & 7 & 13.06 & 0 \\
\hline & 50 & 279 & 0.16 & $40(0)$ & 139 & 717 & 0.11 & $4(0)$ & 127 & 120 & 18.38 & 0 \\
\hline & 60 & 339 & 0.13 & $37(0)$ & 638 & 987 & 0.10 & $9(0)$ & 570 & 543 & 18.25 & 0 \\
\hline & 70 & 399 & 0.11 & $38(0)$ & 698 & 2029 & 0.09 & $17(0)$ & 631 & 572 & 12.69 & 0 \\
\hline & 80 & 459 & 0.06 & $31(0)$ & 459 & 2627 & 0.05 & $38(0)$ & 413 & 382 & 11.66 & 0 \\
\hline & 90 & 519 & 0.08 & $34(0)$ & 1081 & 3822 & 0.07 & $50(0)$ & 962 & 849 & 10.00 & 0 \\
\hline & 100 & 579 & 0.12 & $12(0)$ & 1232 & 7346 & 0.10 & $391(0)$ & 1065 & 877 & 4.57 & 0 \\
\hline \multirow{14}{*}{ 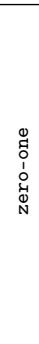 } & 10 & 39 & 1.53 & $40(0)$ & 0 & 26 & 0.00 & $0(0)$ & 0 & 0 & 15.81 & 0 \\
\hline & 15 & 69 & 2.03 & $40(0)$ & 0 & 38 & 0.31 & $0(0)$ & 0 & 0 & 17.27 & 0 \\
\hline & 20 & 99 & 1.11 & $40(0)$ & 0 & 81 & 0.10 & $0(0)$ & 0 & 0 & 12.87 & 0 \\
\hline & 25 & 129 & 0.22 & $40(0)$ & 0 & 116 & 0.09 & $0(0)$ & 0 & 0 & 10.31 & 0 \\
\hline & 30 & 159 & 0.66 & $40(0)$ & 0 & 180 & 0.07 & $0(0)$ & 0 & 0 & 10.64 & 0 \\
\hline & 35 & 189 & 0.19 & $40(0)$ & 0 & 244 & 0.08 & $0(0)$ & 0 & 0 & 8.17 & 0 \\
\hline & 40 & 219 & 0.05 & $40(0)$ & 0 & 375 & 0.05 & $0(0)$ & 0 & 0 & 7.13 & 0 \\
\hline & 45 & 249 & 0.51 & $40(0)$ & 2 & 331 & 0.16 & $0(0)$ & 2 & 0 & 10.17 & 0 \\
\hline & 50 & 279 & 0.22 & $40(0)$ & 6 & 599 & 0.10 & $2(0)$ & 5 & 3 & 9.70 & 0 \\
\hline & 60 & 339 & 0.49 & $40(0)$ & 37 & 1168 & 0.24 & $10(0)$ & 33 & 25 & 13.97 & 0 \\
\hline & 70 & 399 & 0.07 & $40(0)$ & 12 & 2009 & 0.00 & $11(0)$ & 9 & 3 & 3.52 & 0 \\
\hline & 80 & 459 & 0.32 & $40(0)$ & 317 & 3398 & 0.32 & $47(0)$ & 293 & 269 & 14.46 & 0 \\
\hline & 90 & 519 & 0.00 & $40(0)$ & 19 & 2545 & 0.00 & $17(0)$ & 13 & 2 & 2.00 & 0 \\
\hline & 100 & 579 & 0.26 & $31(0)$ & 924 & 14747 & 0.17 & $604(0)$ & 832 & 713 & 6.98 & 0 \\
\hline
\end{tabular}

Table 8: Detailed computational results for the Hose uncertainty set on the PA instances with $a=6$. 


\section{References}

[1] Y. K. Agarwal. k-Partition-based facets of the network design problem. Networks, 47(3):123-139, 2006.

[2] A. Altın, E. Amaldi, P. Belotti, and M. Ç. Pınar. Provisioning virtual private networks under traffic uncertainty. Networks, 49(1):100-115, 2007.

[3] E. Álvarez-Miranda, V. Cacchiani, T. Dorneth, M. Jünger, F. Liers, A. Lodi, T. Parriani, and D. R. Schmidt. Models and algorithms for robust network design with several traffic scenarios. In A. Ridha Mahjoub, V. Markakis, I. Milis, and V. Th. Paschos, editors, ISCO 2012, Revised Selected Papers, volume 7422 of LNCS, pages 261-272. Springer, 2012.

[4] E. Álvarez-Miranda, V. Cacchiani, A. Lodi, T. Parriani, and D. R Schmidt. Singlecommodity robust network design problem: Complexity, instances and heuristic solutions. Technical Report OR-13-14, University of Bologna, 2013.

[5] G. Andreello, A. Caprara, and M. Fischetti. Embedding $\{0,1 / 2\}$-cuts in a branch-and-cut framework: A computational study. INFORMS J. on Computing, 19:229-238, 2007.

[6] A. Atamtürk. On Capacitated Network Design Cut-Set Polyhedra. Math. Program. B, 92(3):425-437, 2002.

[7] P. Avella, S. Mattia, and A. Sassano. Metric Inequalities and the Network Loading Problem. In D. Bienstock and G. Nemhauser, editors, Proceedings of the IPCO, volume 3064 of LNCS, pages 401-421. Springer, 2004.

[8] A.-L. Barabási and R. Albert. Emergence of scaling in random networks. Science, 286(5439):509-512, 1999.

[9] F. Barahona. Network Design Using Cut Inequalities. SIAM J. on Optimization, 6(3):823837, 1996.

[10] W. Ben-Ameur and H. Kerivin. Routing of Uncertain Traffic Demands. Optimization and Engineering, 6(3):283-313, 2005.

[11] A. Ben-Tal and A. Nemirovski. Robust solutions of uncertain linear programs. Oper. Res. Let., 25(1):1-13, 1999.

[12] J. F. Benders. Partitioning procedures for solving mixed-variables programming problems. Numerische Mathematik, 4(1):238-252, 1962.

[13] D. Bertsimas and M. Sim. Robust discrete optimization and network flows. Math. Program. B, 98:49-71, 2003. 
[14] D. Bienstock, S. Chopra, O. Günlük, and C.-Y. Tsai. Minimum cost capacity installation for multicommodity network flows. Math. Program., 81(2):177-199, 1998.

[15] D. Bienstock and O. Günlük. Capacitated Network Design - Polyhedral Structure and Computation. INFORMS J. on Computing, 8(3):243-259, 1996.

[16] C. Buchheim, F. Liers, and L. Sanità. An exact algorithm for robust network design. In J. Pahl, T. Reiners, and S. Voß, editors, Proceedings of the INOC, INOC'11, pages 7-17. Springer, 2011.

[17] A. Caprara and M. Fischetti. \{0, 1/2\}-Chvátal-Gomory cuts. Math. Program., 74(3):221235, 1996.

[18] C. Chekuri, B. F. Shepherd, G. Oriolo, and M. Scutellà. Hardness of robust network design. Networks, 50(1):50-54, 2007.

[19] B. V. Cherkassky and A. V. Goldberg. On implementing push-relabel method for the maximum flow problem. In E. Balas and J. Clausen, editors, Proceedings of the IPCO, volume 920 of $L N C S$, pages 157-171. Springer, 1995.

[20] T. Christof and A. Löbel. PORTA - POlyhedron Representation Transformation Algorithm. http://typo.zib.de/opt-long_projects/Software/Porta/, 2008.

[21] T. Dorneth. Ein Branch-and-Cut-Verfahren für robustes Netzwerkdesign. Diplomarbeit, Universität zu Köln, November 2012.

[22] N. G. Duffield, P. Goyal, A. Greenberg, P. Mishra, K. K. Ramakrishnan, and J. E. van der Merwe. A flexible model for resource management in virtual private networks. In Proceedings of the SIGCOMM, SIGCOMM '99, pages 95-108. ACM, 1999.

[23] J. A. Fingerhut, S. Suri, and J. S. Turner. Designing least-cost nonblocking broadband networks. J. of Algorithms, 24(2):287 - 309, 1997.

[24] L. R. Ford and D. R. Fulkerson. Maximal flow through a network. Canadian J. of Mathematics, 8:399-404, 1956.

[25] A. V. Goldberg and R. E. Tarjan. A new approach to the maximum-flow problem. J. of the ACM, 35(4):921-940, 1988.

[26] R. E. Gomory and T. C. Hu. Synthesis of a Communication Network. J. of the SIAM, 12(2):pp. 348-369, 1964.

[27] D. S. Johnson, M. Minkoff, and S. Phillips. The prize collecting steiner tree problem: Theory and practice. In Proceedings of the SODA, SODA '00, pages 760-769. SIAM, 2000. 
[28] M. Jünger and S. Thienel. The abacus system for branch-and-cut-and-price algorithms in integer programming and combinatorial optimization. Software: Practice and Experience, 30(11):1325-1352, 2000.

[29] R. M. Karp. Reducibility among combinatorial problems. In Complexity of Computer Computations, pages 85-103, 1972.

[30] T. Leighton and S. Rao. Multicommodity max-flow min-cut theorems and their use in designing approximation algorithms. J. of the ACM, 46(6):787-832, 1999.

[31] T. L. Magnanti, P. Mirchandani, and R. Vachani. Modeling and solving the capacitated network loading problem. Technical Report OR-239-91, MIT, 1991.

[32] T. L. Magnanti, P. Mirchandani, and R. Vachani. The convex hull of two core capacitated network design problems. Math. Program., 60(1-3):233-250, 1993.

[33] T. L. Magnanti and R. T. Wong. Accelerating Benders Decomposition: Algorithmic Enhancement and Model Selection Criteria. Oper. Res., 29(3):464-484, 1981.

[34] S. Mattia. The robust network loading problem with dynamic routing. Computational Optimization and Applications, 54:619-643, 2013.

[35] S. T. McCormick, M. R. Rao, and G. Rinaldi. Easy and difficult objective functions for max-cut. Math. Program. B, 94:459-466, 2003.

[36] M. Minoux. Optimum Synthesis of a Network with Non-Simultaneous Multicommodity Flow Requirements. In Annals of Discrete Mathematics (11) Studies on Graphs and Discrete Programming, volume 59, pages 269-277. North-Holland, 1981.

[37] S. Mudchanatongsuk, F. Ordóñez, and J. Liu. Robust solutions for network design under transportation cost and demand uncertainty. J. of the Operational Research Society, 59(5):652-662, 2008.

[38] S. Orlowski, M. Pióro, A. Tomaszewski, and R. Wessäly. SNDlib 1.0-Survivable Network Design Library. In Proceedings of the INOC 2007, 2007. http://sndlib.zib.de, extended version accepted in Networks, 2009.

[39] R. Pesenti, F. Rinaldi, and W. Ukovich. An exact algorithm for the min-cost network containment problem. Networks, 43(2):87-102, 2004.

[40] C. Raack, A. M. C. A. Koster, and R. Wessäly. On the strength of cut-based inequalities for capacitated network design polyhedra. Technical Report 07-08, ZIB, 2007.

[41] L. Sanità. Robust Network Design. Ph.D. Thesis. Università La Sapienza, Roma, 2009. 